\title{
Two families of pro- $p$ groups that are not absolute Galois groups
}

\author{
Claudio Quadrelli* \\ Communicated by Benjamin Klopsch
}

\begin{abstract}
Let $p$ be a prime. We produce two new families of pro- $p$ groups which are not realizable as absolute Galois groups of fields. To prove this, we use the 1-smoothness property of absolute Galois pro- $p$ groups. Moreover, we show in these families, one has several pro- $p$ groups which may not be ruled out as absolute Galois groups employing the quadraticity of Galois cohomology (a consequence of the norm residue theorem), or the vanishing of Massey products in Galois cohomology.
\end{abstract}

\section{Introduction}

Throughout the paper, $p$ will denote a prime number. For a field $\mathbb{K}$, let $\overline{\mathbb{K}}_{S}$ and $\mathbb{K}(p)$ denote respectively the separable closure of $\mathbb{K}$, and the compositum of all finite Galois $p$-extensions of $\mathbb{K}$. The maximal pro- $p$ Galois group of $\mathbb{K}$, denoted by $G_{\mathbb{K}}(p)$, is the maximal pro- $p$ quotient of the absolute Galois group $\operatorname{Gal}\left(\overline{\mathbb{K}}_{s} / \mathbb{K}\right)$ of $\mathbb{K}$, and it coincides with the Galois group of the extension $\mathbb{K}(p) / \mathbb{K}$. Describing maximal pro- $p$ Galois groups of fields among pro- $p$ groups is one of the most important - and challenging - problems in Galois theory (see, e.g., [15, § 3.12], $[21, \S 2.2]$, and $[22, \S 1])$. Already finding explicit examples of pro- $p$ groups which do not occur as maximal pro- $p$ Galois groups - and thus also as absolute Galois groups (see Remark 3.3) - of fields is considered a remarkable achievement (see, e.g., $[2,3,22])$. One of the oldest known obstructions for the realization of a pro- $p$ group as $G_{\mathbb{K}}(p)$ for some field $\mathbb{K}$ is given by the Artin-Schreier theorem: the only finite group realizable as $G_{\mathbb{K}}(p)$ for some field $\mathbb{K}$ is the cyclic group of order 2 (cf. [1]).

In this paper, we produce new examples of torsion-free pro- $p$ groups which do not occur as maximal pro- $p$ Galois groups of fields containing a primitive $p$-th root of 1 , and thus neither as absolute Galois groups. Given two elements $x, y$ of a (pro- $p$ ) group $G$, let $[x, y]=x^{-1} y^{-1} x y$ denote the commutator between $x$ and $y$, and for $n \geq 1$, set

$$
[y, n x]=[\ldots[[y, \underbrace{x], x], \ldots, x}_{n \text { times }}] .
$$


Theorem 1.1. Let $p$ be a prime, and let $G$ be a finitely generated pro- $p$ group, with minimal pro- $p$ presentation

$$
G=\left\langle x_{1}, \ldots, x_{d} \mid r_{0}, r_{1}, \ldots, r_{v}\right\rangle, \quad d \geq 3, v \geq 0,
$$

where either

(i) $r_{0}=x_{1}^{q} \cdot\left[x_{1, n} x_{2}\right] \cdot\left[x_{2}, x_{3}\right] \cdots\left[x_{d-1}, x_{d}\right] \cdot s$, with $d$ odd, or

(ii) $r_{0}=x_{1}^{q} \cdot\left[x_{1, n} x_{2}\right] \cdot\left[x_{3}, x_{4}\right] \cdots\left[x_{d-1}, x_{d}\right] \cdot s$, with $d$ even,

and in both cases, $q=p^{f}$ with $f \in\{1,2, \ldots, \infty\}(f \geq 2$ if $p=2), n \geq 2$, the relations $r_{1}, \ldots, r_{v}$ lie in the closed commutator subgroup $S^{\prime}:=\operatorname{cl}([S, S])$, and $s$ lies in the closed subgroup $\mathrm{cl}\left(\left[S^{\prime}, S^{\prime}\right]\right)$, where $S$ denotes the closed subgroup of $G$ generated by $x_{3}, \ldots, x_{d}$.

Then $G$ does not occur as the maximal pro- $p$ Galois group of a field containing a primitive p-th root of 1 , and thus neither as the absolute Galois group of a field.

To prove Theorem 1.1, we employ the following formal version of Hilbert 90 for pro- $p$ groups, introduced in $[4, \S 14]$ and studied in [12,28,33]. A pro- $p$ group $G$ is said to be 1 -smooth if it may be endowed with a continuous $G$-module $M$, isomorphic to $\mathbb{Z}_{p}$ as an abelian pro- $p$ group, such that, for every open subgroup $U$ of $G$, the canonical map

$$
H^{1}\left(U, M_{U} / p^{n} M_{U}\right) \rightarrow H^{1}\left(U, M_{U} / p M_{U}\right),
$$

induced by the epimorphism of continuous $U$-modules

$$
M_{U} / p^{n} M_{U} \rightarrow M_{U} / p M_{U},
$$

is surjective for every $n \geq 1$ (here $M_{U}$ denotes the restriction of the $G$-module $M$ to $U$ ). By Kummer theory, the maximal pro- $p$ Galois group of a field $\mathbb{K}$ containing a primitive $p$-th root of 1 is 1 -smooth (see Theorem 2.8). We prove that the pro- $p$ groups defined in Theorem 1.1 are not 1-smooth.

After the positive solution of the celebrated Bloch-Kato conjecture given by M. Rost and V. Voevodsky (with a "patch" of C. Weibel, see [37, 40,41]), one knows that the $\mathbb{F}_{p}$-cohomology algebra of the maximal pro- $p$ Galois group $G_{\mathbb{K}}(p)$ of a field $\mathbb{K}$ containing a primitive $p$-th root of 1 ,

$$
H^{\bullet}\left(G_{\mathbb{K}}(p), \mathbb{F}_{p}\right):=\bigoplus_{n \geq 0} H^{n}\left(G_{\mathbb{K}}(p), \mathbb{F}_{p}\right),
$$

with $\mathbb{F}_{p}$ the finite field of $p$ elements considered as a trivial $G_{\mathbb{K}}(p)$-module, and endowed with the cup-product, is a quadratic algebra: i.e., all its elements of positive degree are combinations of products of elements of degree 1, and its defining relations are homogeneous relations of degree 2 (see $\$ 2.2$ ). From this, it 
was possible to obtain new obstructions for the realization of a pro- $p$ group as maximal pro- $p$ Galois group (see, e.g., $[3,27,33,36]$ ). Subsequently, it has been conjectured that the algebra $H^{\bullet}\left(G_{\mathbb{K}}(p), \mathbb{F}_{p}\right)$ has no external operations in addition to its "internal" ring structure with respect to the cup-product: namely, all $n$-fold Massey products of $H^{\bullet}\left(G_{\mathbb{K}}(p), \mathbb{F}_{p}\right)$ vanish (see [23, Conjecture 1.1] and $\S 5.1-5.2)$. This conjecture has been shown to be true for $n=3$ and in other relevant cases (see [11,16-18]).

We show that, from Theorem 1.1, one may produce several one-relator pro$p$ groups (i.e., pro- $p$ groups defined by a single relation) whose $\mathbb{F}_{p}$-cohomology algebra is quadratic and yields the vanishing of 3- and 4-fold Massey products, and which are not absolute Galois groups of fields.

Theorem 1.2. Let $G=\left\langle x_{1}, \ldots, x_{d} \mid r_{0}\right\rangle$ be a one-relator pro- $p$ group with $r_{0}$ as in Theorem 1.1. Then the following hold.

(i) The $\mathbb{F}_{p}$-cohomology algebra $H^{\bullet}\left(G, \mathbb{F}_{p}\right)$ is quadratic.

(ii) (a) If $r_{0}$ is of the first type, then every 3-fold and 4-fold Massey product vanishes in $H^{\bullet}\left(G, \mathbb{F}_{p}\right)$, unless $p=q=3$.

(b) If $r_{0}$ is of the second type, then every 3-fold and 4-fold Massey product vanishes in $H^{\bullet}\left(G, \mathbb{F}_{p}\right)$, unless $p=q=3$ or $n=2,3$.

Therefore, one may not employ these cohomological conditions to rule out the pro- $p$ groups defined in Theorem 1.2 as maximal pro- $p$ Galois groups and absolute Galois groups of fields. Moreover, most of these one-relator pro- $p$ groups do not belong to the family of pro- $p$ groups which are not absolute Galois groups introduced in [2, Corollary 1] (see $\$ 4.3$ ). Hence, Theorems 1.1-1.2 provide a big wealth of genuine new examples of pro- $p$ groups that are not absolute Galois groups of fields - see also Remark 5.11.

The paper is structured as follows. In Section 2, we give basic definitions and properties on the cohomology of pro- $p$ groups (in particular, in Subsection 2.3, we give the definition of 1-smooth pro- $p$ group). In Section 3, we prove Theorem 1.1 (cf. Subsections 3.1-3.2). In Section 4, we prove Theorem 1.2 (i), and we show that our one-relator pro- $p$ groups are different to the pro- $p$ groups defined in [2] (cf. Proposition 4.4). In Section 5, we give a brief (and self-contained) treatment of the group-theoretic interpretation of Massey products in $\mathbb{F}_{p}$-cohomology of pro- $p$ groups (cf. Subsection 5.2), which we use to prove Theorem 1.2 (ii) (a)-(b) (cf. Subsections 5.3-5.4). 


\section{Pro- $p$ groups and Galois cohomology}

We work in the world of pro- $p$ groups. Henceforth, every subgroup of a pro- $p$ group will be tacitly assumed to be closed, and generators of a subgroup will be meant in the topological sense.

In particular, for a pro- $p$ group $G$ and a positive integer $n, G^{n}$ will denote the closed subgroup of $G$ generated by the $n$-th powers of all elements of $G$. Moreover, for two elements $g, h \in G$, we set

$$
h^{g}=g^{-1} h g \quad \text { and } \quad[h, g]=h^{-1} \cdot h^{g},
$$

and for two subgroups $H_{1}, H_{2}$ of $G,\left[H_{1}, H_{2}\right]$ will denote the closed subgroup of $G$ generated by all commutators $[h, g]$ with $h \in H_{1}$ and $g \in H_{2}$. In particular,

(a) $G^{\prime}$ will denote the commutator subgroup $[G, G]$ of $G$;

(b) $\Phi(G)=G^{p} \cdot G^{\prime}$ will denote the Frattini subgroup of $G$;

(c) $\left(\gamma_{n}(G)\right)_{n \geq 1}$ will denote the descending central series of $G$, i.e., $\gamma_{1}(G)=G$, $\gamma_{2}(G)=G^{\prime}$ and $\gamma_{n+1}(G)=\left[\gamma_{n}(G), G\right]$ for $n \geq 2$.

(d) $G_{(3)}$ will denote the subgroup of $G$ defined by

$$
G_{(3)}= \begin{cases}G^{p} \cdot \gamma_{3}(G) & \text { if } p \neq 2, \\ G^{4} \cdot\left(G^{\prime}\right)^{2} \cdot \gamma_{3}(G) & \text { if } p=2,\end{cases}
$$

i.e., $G_{(3)}$ is the third term of the $p$-Zassenhaus filtration of $G$ (cf., e.g., [9, $\S 1]$ ) - note that $\Phi(G) / G_{(3)}$ is a $p$-elementary abelian pro- $p$ group.

Finally, recall that $\mathbb{Z}_{p}$ denotes the ring of $p$-adic integers

$$
\mathbb{Z}_{p}=\left\{a_{0}+a_{1} p+a_{2} p^{2}+\cdots+a_{n} p^{n}+\cdots \mid 0 \leq a_{i} \leq p-1 \text { for all } i \geq 0\right\},
$$

which is a cyclic pro- $p$ group (endowed with the addition), which may be generated by any $p$-adic integer $1+p \lambda, \lambda \in \mathbb{Z}_{p}$.

For further properties of pro- $p$ groups, we refer to [5, Chapters 1-4].

\subsection{Cohomology of pro- $p$ groups}

In this subsection, we focus on finitely generated pro- $p$ groups.

Let $G$ be a pro- $p$ group, and let $\mathbb{F}_{p}$ denote the finite field with $p$-elements. We consider $\mathbb{F}_{p}$ also as a trivial $G$-module. For the zeroth and the first cohomology groups of $G$ with coefficients in $\mathbb{F}_{p}$, we have isomorphisms of $\mathbb{F}_{p}$-vector spaces $H^{0}\left(G, \mathbb{F}_{p}\right) \simeq \mathbb{F}_{p}$ and

$$
H^{1}\left(G, \mathbb{F}_{p}\right)=\operatorname{Hom}_{\mathrm{gp}}\left(G, \mathbb{F}_{p}\right) \simeq(G / \Phi(G))^{*},
$$


where $\operatorname{Hom}_{\mathrm{gp}}\left(,, \mathbb{F}_{p}\right)$ denotes the group of (pro- $p$ ) group homomorphisms to the additive group of $\mathbb{F}_{p}$, and ${ }_{-}^{*}=\operatorname{Hom}_{\mathbb{F}_{p}}\left({ }_{-}, \mathbb{F}_{p}\right)$ denotes the $\mathbb{F}_{p}$-dual (cf. [25, Proposition 3.9.1]). If $\mathcal{X}=\left\{x_{1}, \ldots, x_{d}\right\}$ is a minimal set generating $G$, then by (2.1), one has $d=\operatorname{dim}\left(H^{1}\left(G, \mathbb{F}_{p}\right)\right)$, and $\mathcal{X}$ yields a dual basis $\mathscr{B}=\left\{\chi_{1}, \ldots, \chi_{d}\right\}$ of $H^{1}\left(G, \mathbb{F}_{p}\right)$, i.e., $\chi_{i}\left(x_{j}\right)=\delta_{i j}$ for every $1 \leq i, j \leq d$.

A short exact sequence of pro- $p$ groups $\{1\} \rightarrow N \rightarrow G \rightarrow G / N \rightarrow\{1\}$ induces an exact sequence of $\mathbb{F}_{p}$-vector spaces

$$
\begin{gathered}
0 \longrightarrow H^{1}\left(G / N, \mathbb{F}_{p}\right) \stackrel{\inf _{G / N, G}^{1}}{\longrightarrow} H^{1}\left(G, \mathbb{F}_{p}\right) \stackrel{\operatorname{res}_{G, N}^{1}}{\longrightarrow} H^{1}\left(N, \mathbb{F}_{p}\right)^{G} \longrightarrow \\
\longleftrightarrow H^{2}\left(G / N, \mathbb{F}_{p}\right) \stackrel{\inf _{G / N, G}^{2}}{\longrightarrow} H^{2}\left(G, \mathbb{F}_{p}\right)
\end{gathered}
$$

(cf. [25, Proposition 1.6.7]).

A short exact sequence of pro- $p$ groups

$$
\{1\} \rightarrow R \rightarrow F \rightarrow G \rightarrow\{1\},
$$

with $F$ a free pro- $p$ group, is called a minimal presentation if $R \subseteq \Phi(F)$ or, equivalently, if the map $\inf _{G, F}^{1}$ induced by the epimorphism $F \rightarrow G$ is an isomorphism. Moreover, since $H^{2}\left(F, \mathbb{F}_{p}\right)=0$ (cf. [25, Proposition 3.9.5]), the map trg induces an isomorphism of $\mathbb{F}_{p}$-vector spaces

$$
\operatorname{trg}^{-1}: H^{2}\left(G, \mathbb{F}_{p}\right) \stackrel{\sim}{\rightarrow} H^{1}\left(R, \mathbb{F}_{p}\right)^{F} \simeq\left(R / R^{p}[F, R]\right)^{*} .
$$

If $\left\{r_{i}, i \in \mathcal{Q}\right\} \subseteq R$ is a minimal set generating $R$ as normal subgroup of $F$ and if $\mathcal{X}=\left\{x_{1}, \ldots, x_{d}\right\}$ is a minimal set generating $G$, we write

$$
G=\left\langle x_{1}, \ldots, x_{d} \mid r_{i}, i \in \mathcal{d}\right\rangle,
$$

and by (2.4), one has $\operatorname{dim}\left(H^{2}\left(G, \mathbb{F}_{p}\right)\right)=|\ell|$. A pro- $p$ group $G$ with minimal presentation (2.3) is a one-relator pro- $p$ group if $R$ is generated as normal subgroup of $F$ by a single element $r$, i.e., if $\operatorname{dim}\left(H^{2}\left(G, \mathbb{F}_{p}\right)\right)=1$.

The $\mathbb{F}_{p}$-cohomology of a pro- $p$ group comes endowed with the cup-product

$$
H^{s}\left(G, \mathbb{F}_{p}\right) \times H^{t}\left(G, \mathbb{F}_{p}\right) \stackrel{\cup}{\rightarrow} H^{s+t}\left(G, \mathbb{F}_{p}\right), \quad s, t \geq 0,
$$

which is graded-commutative, i.e.,

$$
\beta \cup \alpha=(-1)^{s t} \alpha \cup \beta \quad \text { for } \alpha \in H^{s}\left(G, \mathbb{F}_{p}\right), \beta \in H^{t}\left(G, \mathbb{F}_{p}\right)
$$

(cf. [25, Chapter I, § 4]).

For finitely generated one-relator pro- $p$ groups, one has the following (cf. [30, Propositions 4.2 and 4.6]). 
Proposition 2.1. Let $G=\left\langle x_{1}, \ldots, x_{d} \mid r\right\rangle$ be a finitely generated one-relator pro- $p$ group. If

$$
r \equiv\left[x_{1}, x_{2}\right] \cdot\left[x_{3}, x_{4}\right] \cdots\left[x_{n-1}, x_{n}\right] \bmod G_{(3)},
$$

for some $n$ even, with $2 \leq n \leq d$, then $H^{\bullet}\left(G, \mathbb{F}_{p}\right)$ is quadratic. In particular, if $\mathscr{B}=\left\{\chi_{1}, \ldots, \chi_{d}\right\}$ is a basis of $H^{1}\left(G, \mathbb{F}_{p}\right)$ dual to $\mathcal{X}=\left\{x_{1}, \ldots, x_{d}\right\}$, then one has

$$
\begin{aligned}
& \chi_{1} \cup \chi_{2}=\chi_{3} \cup \chi_{4}=\cdots=\chi_{n-1} \cup \chi_{n}, \\
& \chi_{i} \cup \chi_{j}=0 \quad \text { for } 1 \leq i \leq j \leq d,(i, j) \neq(1,2), \ldots(n-1, n),
\end{aligned}
$$

and $\chi_{1} \cup \chi_{2}$ generates $H^{1}\left(G, \mathbb{F}_{p}\right)$. Moreover, $H^{k}\left(G, \mathbb{F}_{p}\right)=0$ for $k \geq 3$.

One-relator pro- $p$ groups satisfying (2.5) generalize Demushkin groups, which were studied by S. P. Demuškin, J.-P. Serre and J.P. Labute (cf., e.g., [25, Chapter III, pp. 231-244]) - in fact, Proposition 2.1 is based on the description of the cup-product for Demushkin groups.

For further facts on cohomology of pro- $p$ groups, we direct the reader to [34, Chapter I, § 3-4] and to [25, Chapter III, § 9].

\subsection{Quadratic cohomology and the norm residue theorem}

Let $G$ be a pro- $p$ group. The $\mathbb{F}_{p}$-cohomology

$$
H^{\bullet}\left(G, \mathbb{F}_{p}\right)=\bigoplus_{n \geq 0} H^{n}\left(G, \mathbb{F}_{p}\right)
$$

endowed with the cup-product, is a non-negatively graded algebra over $\mathbb{F}_{p}$. A special class of non-negatively graded $\mathbb{F}_{p}$-algebras is given by the following (cf. [26]).

Definition 2.2. Let $A_{\bullet}=\bigoplus_{n \geq 0} A_{n}$ be a (non-negatively) graded $\mathbb{F}_{p}$-algebra, with $A_{0}=\mathbb{F}_{p}$. Then $A_{\bullet}$ is said to be quadratic if one has an isomorphism of graded algebras

$$
A_{\bullet} \simeq \frac{T_{\bullet}\left(A_{1}\right)}{(\Omega)},
$$

where $T_{\bullet}\left(A_{1}\right)=\bigoplus_{n \geq 0} A_{1}^{\otimes n}$ denotes the $\mathbb{F}_{p}$-tensor algebra generated by $A_{1}$, and $(\Omega)$ is the two-sided ideal of $T_{\bullet}\left(A_{1}\right)$ generated by $\Omega$, with $\Omega \subseteq A_{1} \otimes A_{1}$.

Example 2.3. Let $V$ be a finite-dimensional $\mathbb{F}_{p}$-vector space. Then the symmetric algebra $S_{\bullet}(V)$ and the exterior algebra $\bigwedge_{\bullet}(V)$ are quadratic algebras. 
A consequence of the norm residue theorem is that the $\mathbb{F}_{p}$-cohomology algebra of the maximal pro- $p$ Galois group of a field containing a primitive $p$-th root of 1 is quadratic (cf., e.g., [8, § 24.3] and [27, § 2]).

Theorem 2.4. Let $\mathbb{K}$ be a field containing a primitive $p$-th root of 1 . Then the $\mathbb{F}_{p}$-cohomology algebra $H^{\bullet}\left(G_{\mathbb{K}}(p), \mathbb{F}_{p}\right)$ is a quadratic algebra.

A pro- $p$ group $G$ such that the $\mathbb{F}_{p}$-cohomology algebra $H^{\bullet}\left(H, \mathbb{F}_{p}\right)$ is quadratic for every subgroup $H \subseteq G$ is called a Bloch-Kato pro-p group (cf. [27]). By Theorem 2.4, the maximal pro- $p$ Galois group of a field $\mathbb{K}$ containing a primitive $p$-th root of 1 is Bloch-Kato, as every subgroup $H \subseteq G_{\mathbb{K}}(p)$ is the maximal pro- $p$ Galois group of an extension of $\mathbb{K}$.

Remark 2.5. If $p \neq 2$ and $G$ is a finitely generated Bloch-Kato pro- $p$ group, then

$$
\operatorname{dim}\left(H^{2}\left(G, \mathbb{F}_{p}\right)\right) \leq\left(\begin{array}{c}
\operatorname{dim}\left(H^{1}\left(G, \mathbb{F}_{p}\right)\right) \\
2
\end{array}\right)
$$

(cf. [27, §4.1]). Thus, if $G$ is as in Theorem 1.1 and $v>\left(\begin{array}{l}d \\ 2\end{array}\right)$, then $G$ is not BlochKato.

By results of Ware (cf. [39]) and Engler, Koenigsmann and Nogueira (cf. [13, $14]$ ), if $\mathbb{K}$ is a field containing a primitive $p$-th root of 1 , then the maximal pro- $p$ Galois group $G_{\mathbb{K}}(p)$ has the following properties: (i) if $G_{\mathbb{K}}(p)$ is not meta-abelian (i.e., $G_{\mathbb{K}}(p)^{\prime}$ is abelian), then $G_{\mathbb{K}}(p)$ contains a free non-abelian subgroup; and (ii) $G_{\mathbb{K}}(p)$ contains a unique maximal abelian normal subgroup $A$, and

$$
G_{\mathbb{K}}(p) \simeq A \rtimes\left(G_{\mathbb{K}}(p) / A\right) .
$$

The following result extends these properties of maximal pro- $p$ Galois groups to Bloch-Kato pro- $p$ groups (cf. [27, Theorem B] and [33, Theorem 1.2]).

Proposition 2.6. Let $G$ be a Bloch-Kato pro-p group.

(i) If $G$ is not meta-abelian, then $G$ contains a free non-abelian subgroup.

(ii) If $G$ is also 1-smooth, then $G$ contains a unique maximal abelian subgroup $A$, and $G \simeq A \rtimes G / A$.

\subsection{Kummerian pro- $p$ pairs}

Let $1+p \mathbb{Z}_{p}=\left\{1+p \lambda \mid \lambda \in \mathbb{Z}_{p}\right\}$ denote the pro- $p$ Sylow subgroup of the group of units of the ring of $p$-adic integers $\mathbb{Z}_{p}$. A pair $\mathcal{E}=(G, \theta)$ consisting of a pro- $p$ group and a continuous homomorphism $\theta: G \rightarrow 1+p \mathbb{Z}_{p}$ is called a cyclotomic 
pro- $p$ pair, and the morphism $\theta$ is called an orientation of $G$ (cf. [7, §3] and [33]). A cyclotomic pro- $p$ pair $\mathcal{E}=(G, \theta)$ is said to be torsion-free if $p$ is odd, or if $p=2$ and $\operatorname{Im}(\theta) \subseteq 1+4 \mathbb{Z}_{2}$. (Note that the pro- $p$ group $G$ might have non-trivial torsion even though $\mathcal{E}=(G, \theta)$ is a torsion-free cyclotomic pro- $p$ pair.) Given a cyclotomic pro- $p$ pair $\mathcal{G}=(G, \theta)$, one has the following constructions:

(a) if $H$ is a subgroup of $G$, we set $\left.\mathscr{E}\right|_{H}:=\left(H,\left.\theta\right|_{H}\right)$;

(b) if $N$ is a normal subgroup of $G$ contained in $\operatorname{Ker}(\theta)$, then $\theta$ induces an orientation $\bar{\theta}: G / N \rightarrow 1+p \mathbb{Z}_{p}$, and we set $\mathscr{E} / N:=(G / N, \bar{\theta})$.

Given a cyclotomic pro- $p$ pair $\mathscr{E}=(G, \theta)$, the pro- $p$ group $G$ comes endowed with a distinguished subgroup $K(\mathscr{G})$, introduced in $[12, \S 3]$ and defined by

$$
K(\mathscr{G})=\left\langle h^{-\theta(g)} \cdot h^{g^{-1}} \mid g \in G, h \in \operatorname{Ker}(\theta)\right\rangle .
$$

The subgroup $K(\mathscr{G})$ is normal in $G$, and it is contained in $\operatorname{Ker}(\theta)$. Moreover, one has $K(\mathscr{E}) \supseteq \operatorname{Ker}(\theta)^{\prime}$ and $K(\mathscr{E}) \subseteq \Phi(G)$. Thus, the quotient $\operatorname{Ker}(\theta) / K(\mathscr{E})$ is abelian.

Definition 2.7. Given a cyclotomic pro- $p$ pair $\mathscr{E}=(G, \theta)$, let $\mathbb{Z}_{p}(\theta)$ denote the continuous $G$-module of rank 1 induced by $\theta$, i.e., $\mathbb{Z}_{p}(\theta) \simeq \mathbb{Z}_{p}$ as abelian pro- $p$ groups, and $g . \lambda=\theta(g) \cdot \lambda$ for every $\lambda \in \mathbb{Z}_{p}(\theta)$. The pair $\mathcal{G}$ is said to be Kummerian if, for every $n \geq 1$, the map

$$
H^{1}\left(G, \mathbb{Z}_{p}(\theta) / p^{n} \mathbb{Z}_{p}(\theta)\right) \rightarrow H^{1}\left(G, \mathbb{F}_{p}\right),
$$

induced by the epimorphism of $G$-modules

$$
\mathbb{Z}_{p}(\theta) / p^{n} \mathbb{Z}_{p}(\theta) \rightarrow \mathbb{Z}_{p}(\theta) / p \mathbb{Z}_{p}(\theta) \simeq \mathbb{F}_{p},
$$

is surjective. Moreover, $\mathscr{E}$ is 1 -smooth if $\left.\mathscr{E}\right|_{H}$ is Kummerian for every subgroup $H \subseteq G$.

We say that a pro- $p$ group $G$ may complete into a Kummerian, or 1-smooth, pro- $p$ pair if there exists an orientation $\theta: G \rightarrow 1+p \mathbb{Z}_{p}$ such that the pair $(G, \theta)$ is Kummerian, respectively 1 -smooth.

Given a field $\mathbb{K}$ containing a primitive $p$-th root of 1 , let $\theta_{\mathbb{K}}: G_{\mathbb{K}}(p) \rightarrow 1+p \mathbb{Z}_{p}$ denote the pro- $p$ cyclotomic character, i.e., if $\zeta$ is a primitive $p^{k}$-th root of 1 , with $k \geq 1$, then $\sigma(\zeta)=\zeta^{\theta_{\mathbb{K}}(\sigma)}$ for all $\sigma \in G_{\mathbb{K}}(p)$ (cf., e.g., [12, § 4]). The continuous $G_{\mathbb{K}}(p)$-module $\mathbb{Z}_{p}\left(\theta_{\mathbb{K}}\right)$ is called the first Tate twist module, and usually, it is denoted by $\mathbb{Z}_{p}(1)$ (cf. [25, Definition 7.3.6]). By Kummer theory, one has the following (cf. [12, Theorem 4.2] and [33, Proposition 2.3]). 
Theorem 2.8. Let $\mathbb{K}$ be a field containing a primitive $p$-th root of 1 . Then the cyclotomic pro-p pair $\mathscr{E}_{\mathbb{K}}=\left(G_{\mathbb{K}}(p), \theta_{\mathbb{K}}\right)$ is 1-smooth.

Kummerian pro- $p$ pairs and 1-smooth pro- $p$ pairs were introduced in [12] and in $[4, \S 14]$ respectively. In [33], if $\mathscr{G}=(G, \theta)$ is a 1 -smooth pro- $p$ pair, the orientation $\theta$ is said to be 1 -cyclotomic.

Remark 2.9. In $[4, \S 14.1]$, a pro- $p$ pair is defined to be 1 -smooth if the maps (2.6) are surjective for every open subgroup of $G$, yet by a limit argument, this implies also that the maps (2.6) are surjective also for every closed subgroup of $G$ (cf. [33, Corollary 3.2]).

Henceforth, we will restrict our attention to torsion-free cyclotomic pro- $p$ pairs whose pro- $p$ group is finitely generated. One has the following group-theoretic characterization of Kummerianity (cf. [12, Theorems 5.6 and 7.1]).

Theorem 2.10. Let $\mathcal{E}=(G, \theta)$ be a torsion-free cyclotomic pro-p pair, with $G$ finitely generated. The following are equivalent.

(i) The pair $\mathscr{E}$ is Kummerian.

(ii) The quotient $\operatorname{Ker}(\theta) / K(\mathscr{E})$ is a free abelian pro- $p$ group.

(iii) The pair $\mathscr{G} / N$ is Kummerian for every normal subgroup $N$ of $G$ contained in $K(\mathscr{Y})$.

Remark 2.11. Let $\mathscr{E}=(G, \theta)$ be a cyclotomic pro- $p$ pair with $\theta \equiv \mathbf{1}$, i.e., $\theta$ is constantly equal to 1 . Since $K(\mathscr{G})=G^{\prime}$ in this case, by Theorem 2.10, the pair $\mathcal{E}$ is Kummerian if and only if the quotient $G / G^{\prime}$ is torsion-free. Moreover, $\mathscr{E}$ is 1 -smooth if and only if $H / H^{\prime}$ is torsion-free for every subgroup $H \subseteq G$. Pro- $p$ groups with such a property are called absolutely torsion-free, and they were introduced and studied by Würfel in [42].

Examples 2.12. (a) A cyclotomic pro- $p$ pair $(G, \theta)$ with $G$ a free pro- $p$ group is 1 -smooth for any orientation $\theta: G \rightarrow 1+p \mathbb{Z}_{p}$ (cf. [33, § 2.2]).

(b) A cyclotomic pro- $p$ pair $(G, \theta)$ with $G$ an infinite Demushkin pro- $p$ group is 1 -smooth if and only if $\theta: G \rightarrow 1+p \mathbb{Z}_{p}$ is the canonical orientation of $G$, defined as in [20, Theorem 4] (cf. [12, Theorem 7.6]).

(c) The only 1-smooth pro- $p$ pair $(G, \theta)$ with $G$ a finite $p$-group is the cyclic group of order $2 G \simeq \mathbb{Z} / 2$, endowed with the only non-trivial orientation

$$
\theta: G \rightarrow\{ \pm 1\} \subseteq 1+2 \mathbb{Z}_{2}
$$

(cf. [12, Example 3.5]). 
Remark 2.13. By Example 2.12 (c), if $\mathscr{E}=(G, \theta)$ is a torsion-free 1-smooth pro- $p$ pair (i.e., $\operatorname{Im}(\theta)$ is torsion-free), then $G$ is a torsion-free pro- $p$ group.

Kummerianity is inherited by certain quotients of pro- $p$ pairs (cf. [29, Theorem 1.1]).

Proposition 2.14. Let $\mathcal{E}=(G, \theta)$ be a torsion-free Kummerian pro-p pair, an let $N$ be a normal subgroup of $G$ satisfying

(i) $G / N$ is a finitely generated pro- $p$ group,

(ii) $N \subseteq \operatorname{Ker}(\theta)$,

(iii) the map $\operatorname{res}_{G, N}^{1}: H^{1}\left(G, \mathbb{F}_{p}\right) \rightarrow H^{1}\left(N, \mathbb{F}_{p}\right)^{G}$ is surjective.

Then the pro-p pair $\mathscr{G} / N$ is Kummerian.

Remark 2.15. By (2.2), condition (iii) of Proposition 2.14 amounts to requiring that the inclusion $N \subseteq G$ induces a monomorphism of $p$-elementary abelian pro- $p$ groups $N / N^{p}[G, N] \hookrightarrow G / \Phi(G)$.

Let $\mathscr{E}=(G, \theta)$ be a cyclotomic pro- $p$ pair. A continuous 1 -cocycle from $G$ to the $G$-module $\mathbb{Z}_{p}(\theta)$ is a continuous map $c: G \rightarrow \mathbb{Z}_{p}(\theta)$ satisfying

$$
c(x y)=c(x)+\theta(x) \cdot c(y) \text { for every } x, y \in G
$$

(cf., e.g., [25, Chapter I, § 2]). One may employ continuous 1-cocycles to check whether a given cyclotomic pro- $p$ pair $\mathscr{E}$ is Kummerian (cf. [20, Proposition 6]).

Proposition 2.16. Let $\mathscr{G}=(\mathscr{E}, \theta)$ be a torsion-free cyclotomic pro-p pair with $G$ finitely generated, and let $\mathcal{X}=\left\{x_{1}, \ldots, x_{d}\right\}$ be a minimal generating set of $G$. Then $\mathcal{E}$ is Kummerian if and only if, for any sequence $\left(\lambda_{1}, \ldots, \lambda_{d}\right)$ with $\lambda_{i} \in \mathbb{Z}_{p}$ for every $i=1, \ldots, d$, there exists a continuous 1-cocycle $c: G \rightarrow \mathbb{Z}_{p}(\theta)$ such that $c\left(x_{i}\right)=\lambda_{i}$ for every $i=1, \ldots, d$.

Examples 2.17. (a) For $p \neq 2$, let $G$ be the pro- $p$ group with minimal presentation

$$
G=\left\langle x, y, z \mid[x, y]=z^{p}\right\rangle,
$$

and let $\theta: G \rightarrow 1+p \mathbb{Z}_{p}$ be an arbitrary orientation. Then there are no continuous 1-cocycles $c: G \rightarrow \mathbb{Z}_{p}(\theta)$ satisfying $c(x)=c(y)=0$ and $c(z)=1$, and thus the pro- $p$ pair $(G, \theta)$ is not Kummerian for any orientation $\theta$ (cf. [12, Theorem 8.1]). 
(b) Let

$$
H=\left\{\left(\begin{array}{lll}
1 & a & c \\
0 & 1 & b \\
0 & 0 & 1
\end{array}\right) \mid a, b, c \in \mathbb{Z}_{p}\right\}
$$

be the Heisenberg pro- $p$ group. By Remark 2.11, the pair $(H, \mathbf{1})$ is Kummerian, as $H / H^{\prime} \simeq \mathbb{Z}_{p}^{2}$, but $H$ is not absolutely torsion-free. In particular, $H$ contains an open subgroup $U$ which is isomorphic to the pro- $p$ group $G$ as in (a), and therefore $H$ cannot complete into a 1-smooth pro- $p$ pair (cf. [28, Example 5.4]).

\section{Pro- $p$ groups that are not absolute Galois groups}

Let $x, y, z$ be elements of a (pro- $p$ ) group $G$, and $n \geq 1$. Recall that, by commutator calculus, one has the equalities

$$
\begin{aligned}
& {[x y, z]=[x, z]^{y} \cdot[y, z], \quad[x, y z]=[x, z] \cdot[x, y]^{z},} \\
& {\left[x^{n}, y\right]=[x, y]^{x^{n-1}} \cdots[x, y]^{x} \cdot[x, y], \quad\left[x, y^{n}\right]=[x, y] \cdot[x, y]^{y} \cdots[x, y]^{y^{n-1}} \text {. }}
\end{aligned}
$$

Lemma 3.1. Let $x, y$ be elements of a (pro-p) group $G$. For $n \geq 1$, one has the following:

$$
\left[y, x^{n}\right] \equiv \prod_{i=1}^{n}\left[y,{ }_{i} x\right]^{\left(\begin{array}{c}
n \\
i
\end{array}\right)} \bmod G^{\prime \prime},
$$

where $G^{\prime \prime}$ denotes the commutator subgroup of $G^{\prime}$.

Proof. We proceed by induction on $n$. If $n=2$, then

$$
\left[y, x^{2}\right]=[y, x] \cdot[y, x]^{x}=[y, x]^{2}[y, 2 x] .
$$

Now assume that (3.1) holds for $n-1$. Then

$$
\begin{aligned}
& {\left[y, x^{n}\right]=[y, x]\left[y, x^{n-1}\right]^{x}=[y, x]\left[y, x^{n-1}\right]\left[\left[y, x^{n-1}\right], x\right]} \\
& \equiv[y, x] \cdot \prod_{i=1}^{n-1}\left[y,{ }_{i} x\right]^{\left(\begin{array}{c}
n-1 \\
i
\end{array}\right)} \cdot\left[\prod_{i=1}^{n-1}[y, i x]^{\left(\begin{array}{c}
n-1 \\
i
\end{array}\right)}, x\right] \bmod G^{\prime \prime}
\end{aligned}
$$

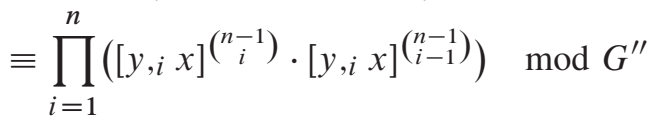

(here we set implicitly $\left(\begin{array}{c}n-1 \\ n\end{array}\right)=0$ ), and the well-known equality

$$
\left(\begin{array}{c}
n-1 \\
i
\end{array}\right)+\left(\begin{array}{c}
n-1 \\
i-1
\end{array}\right)=\left(\begin{array}{l}
n \\
i
\end{array}\right)
$$

yields (3.1). 
Also, we recall the following (cf. [12, Lemma 6.1]).

Lemma 3.2. Let $\mathcal{E}=(G, \theta)$ be a torsion-free cyclotomic pro-p pair, and suppose that $c: G \rightarrow \mathbb{Z}_{p}(\theta)$ is a continuous 1-cocycle. Then, for every $x, y \in G$ and $\lambda \in \mathbb{Z}_{p}$, one has

$$
\begin{aligned}
c\left(x^{\lambda}\right) & = \begin{cases}\lambda \cdot c(x) & \text { if } \theta(x)=1, \\
\frac{\theta(x)^{\lambda}-1}{\theta(x)-1} \cdot c(x) & \text { if } \theta(x) \neq 1 ;\end{cases} \\
c([x, y]) & =\theta(x y)^{-1}((1-\theta(y)) c(x)-(1-\theta(x)) c(y)) .
\end{aligned}
$$

In particular, $c(1)=0$, and for $n \geq 1$, one has

$$
c([y, n x])=\left(\theta(x)^{-1}-1\right)^{n-1} c([y, x]) .
$$

Finally, we underline the following straightforward Galois-theoretic observation.

Remark 3.3. Let $\mathbb{K}$ be a field. If $p=2$, then clearly $\mathbb{K}$ contains the primitive second root of 1 , i.e., -1 ; on the other hand, if $p \neq 2$ and $\mathbb{K}$ does not contain a primitive $p$-th root of 1 , say $\zeta_{p} \in \overline{\mathbb{K}}_{s}$, then $\left[\mathbb{K}\left(\zeta_{p}\right): \mathbb{K}\right]=p-1$, and the absolute Galois group $\mathrm{Gal}\left(\overline{\mathbb{K}}_{S} / \mathbb{K}\right)$ is not a pro- $p$ group. Therefore, if $\mathrm{Gal}\left(\overline{\mathbb{K}}_{s} / \mathbb{K}\right)$ is a pro- $p$ group, then $\zeta_{p} \in \mathbb{K}$.

This yields the following: if a pro- $p$ group does not occur as maximal pro- $p$ Galois group of a field containing a primitive $p$-th root of 1 , then it does not occur as absolute Galois group of any field.

\subsection{The first type of $\boldsymbol{r}_{\mathbf{0}}$}

In this subsection, $G$ denotes a pro- $p$ group with minimal presentation

$$
G=\left\langle x_{1}, \ldots, x_{d} \mid r_{0}, r_{1} \ldots, r_{v}\right\rangle
$$

as in Theorem 1.1, with $r_{0}$ of the first type, i.e., $d$ is odd and

$$
r_{0}=x_{1}^{q} \cdot\left[x_{1, n} x_{2}\right] \cdot\left[x_{2}, x_{3}\right] \cdots\left[x_{d-1}, x_{d}\right] \cdot s, \quad \text { with } s \in S^{\prime \prime},
$$

where $S$ is the subgroup of $G$ generated by $x_{3}, \ldots, x_{d}$, while $r_{1}, \ldots, r_{v} \in S^{\prime}$. Our goal is to show that, for any orientation $\theta: G \rightarrow 1+p \mathbb{Z}_{p}$, the pro- $p$ pair $\mathcal{E}=(G, \theta)$ is not 1 -smooth.

First, we establish what a suitable orientation $\theta: G \rightarrow 1+p \mathbb{Z}_{p}$ should be like. 
Proposition 3.4. Let $\theta: G \rightarrow 1+p \mathbb{Z}_{p}$ be an orientation of $G$ such that the cyclotomic pro-p pair $\mathscr{E}=(G, \theta)$ is torsion-free. If $\mathscr{E}$ is Kummerian, then $q=0$ and $\theta\left(x_{i}\right)=1$ for $i=2, \ldots, d$.

Proof. First, note that $\left.\theta\right|_{S^{\prime}} \equiv \mathbf{1}$, and thus, by Lemma 3.2, one has $\left.c\right|_{S^{\prime \prime}} \equiv \mathbf{0}$ for every continuous 1 -cocycle $c: G \rightarrow \mathbb{Z}_{p}(\theta)$. From

$$
1=\theta(1)=\theta\left(x_{1}^{q}\left[x_{1, n} x_{2}\right]\left[x_{3}, x_{4}\right] \cdots\left[x_{d-1}, x_{d}\right] \cdot s\right)=\theta\left(x_{1}\right)^{q}
$$

we deduce $\theta\left(x_{1}\right)^{q}=1$. Since $\operatorname{Im}(\theta)$ is torsion-free, if $q \neq 0$, one has $\theta\left(x_{1}\right)=1$, while one might have $\theta\left(x_{1}\right) \neq 1$ only if $q=0$.

For every $i=1, \ldots, d$, let $c_{i}: G \rightarrow \mathbb{Z}_{p}(\theta)$ be the continuous 1-cocycle such that $c_{i}\left(x_{j}\right)=\delta_{i j}$, with $j \in\{1, \ldots, d\}$ (such a continuous 1-cocycle always exists by Proposition 2.16). Then

$$
\begin{aligned}
0 & =c_{i}\left(x_{1}^{q}\left[x_{1, n} x_{2}\right]\left[x_{2}, x_{3}\right] \cdots\left[x_{d-1}, x_{d}\right] \cdot s\right) \\
& =c_{i}\left(x_{1}^{q}\right)+\theta\left(x_{1}\right)^{q} \cdot c_{i}\left(\left[x_{1}, n x_{2}\right]\left[x_{3}, x_{4}\right] \cdots\left[x_{d-1}, x_{d}\right] \cdot s\right) \\
& =c_{i}\left(x_{1}^{q}\right)+c_{i}\left(\left[x_{1, n} x_{2}\right]\right)+c_{i}\left(\left[x_{3}, x_{4}\right]\right)+\cdots+c_{i}\left(\left[x_{d-1}, x_{d}\right]\right) .
\end{aligned}
$$

For $i \geq 4, i$ even, from (2.7), Lemma 3.2, and (3.2), we get

$$
0=0+\cdots+c_{i}\left(\left[x_{i}, x_{i+1}\right]\right)+\cdots+0=\theta\left(x_{i} x_{i+1}\right)^{-1}\left(1-\theta\left(x_{i+1}\right)\right) \cdot 1,
$$

and hence $\theta\left(x_{i+1}\right)=1$. Analogously, for $i \geq 3, i$ odd, one gets

$$
0=0+\cdots+c_{i}\left(\left[x_{i-1}, x_{i}\right]\right)+\cdots+0=\theta\left(x_{i-1} x_{i}\right)^{-1}\left(-1+\theta\left(x_{i-1}\right)\right) \cdot 1,
$$

and hence $\theta\left(x_{i-1}\right)=1$. For $i=2$, from (2.7), Lemma 3.2, and (3.2), we get

$$
\begin{aligned}
0 & =0+c_{2}\left(\left[x_{1, n} x_{2}\right]\right)+c_{2}\left(\left[x_{2}, x_{3}\right]\right)+\cdots+0 \\
& =-\theta\left(x_{1}\right)\left(\theta\left(x_{2}\right)^{-1}-1\right)^{n}+\theta\left(x_{2} x_{3}\right)^{-1}\left(1-\theta\left(x_{3}\right)\right) \\
& =-\theta\left(x_{1}\right)\left(\theta\left(x_{2}\right)^{-1}-1\right)^{n}+0,
\end{aligned}
$$

as $\theta\left(x_{3}\right)=1$, and hence $\theta\left(x_{2}\right)=1$. Finally, for $i=1$, from (2.7), Lemma 3.2, and (3.2), we get

$$
0=c_{1}\left(x_{1}^{q}\right)+c_{1}\left(\left[x_{1, n} x_{2}\right]\right)+0= \begin{cases}q & \text { if } \theta\left(x_{1}\right)=1, \\ \frac{\theta\left(x_{1}\right)^{q}-1}{\theta\left(x_{1}\right)-1} & \text { if } \theta\left(x_{1}\right) \neq 1 .\end{cases}
$$

Therefore, $q=0$, and one might have $x_{1} \notin \operatorname{Ker}(\theta)$. 
Note that if $\mathscr{E}=(G, \theta)$ is a Kummerian pro- $p$ pair, then $S \subseteq \operatorname{Ker}(\theta)$, and thus Lemma 3.2 implies that $\left.c\right|_{S^{\prime}} \equiv \mathbf{0}$ for every continuous 1 -cocycle $c: G \rightarrow \mathbb{Z}_{p}(\theta)$. From now on, we assume that $q=0$ so that $G$ may be completed into a Kummerian pro- $p$ pair $\mathscr{E}=(G, \theta)$.

Let $\phi: G \rightarrow \mathbb{Z} / p \mathbb{Z}$ be the homomorphism of pro- $p$ groups defined by $x_{i} \mapsto 0$ for $i=1,3,4, \ldots, d$ and $x_{2} \mapsto 1$, and set $U=\operatorname{Ker}(\phi)$.

Lemma 3.5. The set

$$
\mathcal{X}_{U}=\left\{\begin{array}{c}
t:=x_{2}^{p}, \\
x_{1},\left[x_{1}, x_{2}\right], \ldots,\left[x_{1, l-1} x_{2}\right], \\
x_{3},\left[x_{3}, x_{2}\right], \ldots,\left[x_{3}, l^{\prime}-1 x_{2}\right], \\
x_{i},\left[x_{i}, x_{2}\right], \ldots,\left[x_{i, p-1} x_{2}\right], \quad i=4, \ldots, d
\end{array}\right\},
$$

where $l=\min \{n, p\}$, and $l^{\prime}=p-n$ if $n<p, l^{\prime}=1$ if $n \geq p$, is a minimal generating set of $U$.

Proof. Set $\bar{U}=U / U^{\prime}$, and for $g \in U$, let $\bar{g}=g \cdot U^{\prime}$ denote its image in $\bar{U}$. In particular, we set $v_{i, 0}=\bar{x}_{i}$ and $\left.v_{i, k}=\overline{\left[x_{i}, k\right.} x_{2}\right] \in \bar{U}$ for $i=1, \ldots, d$ and $k \geq 1$.

The group $U$ is generated as normal subgroup of $G$ by $t$ and $x_{1}, x_{3}, x_{4}, \ldots, x_{d}$. Since $G^{\prime} \subseteq U$, one has $G^{\prime \prime} \subseteq U^{\prime}$. Also, $S^{\prime} \subseteq U^{\prime}$, as $S \subseteq U$. By Lemma 3.1, for every $i=1,3, \ldots, d$, one has

$$
\left[x_{i}, t\right] \equiv\left(\prod_{k=1}^{p-1}\left[x_{i}, k x_{2}\right]^{\left(\begin{array}{l}
p \\
k
\end{array}\right)}\right) \cdot\left[x_{i, p} x_{2}\right] \bmod U^{\prime},
$$

and since $\left[x_{i}, t\right] \in U^{\prime}$, it follows that, for every $k \geq p$,

$$
v_{i, k}=v_{i, 1}^{\lambda_{1}} \cdot v_{i, 2}^{\lambda_{2}} \cdots v_{i, p-2}^{\lambda_{p-2}} \cdot v_{i, p-1}^{\lambda_{p-1}}
$$

for some $\lambda_{1}, \ldots, \lambda_{p-1} \in p \mathbb{Z}_{p}$. Hence, $\bar{U}$ is generated as abelian pro- $p$ group by the set $\left\{\bar{t}, v_{i, k}\right\}_{i, k}$, with $i=1,3, \ldots, d$ and $0 \leq k \leq p-1$.

In $U$, from the defining relation $r_{0}=1$, one obtains $p$ relations

$$
\begin{aligned}
& r_{0}=\left[x_{1, n} x_{2}\right]\left[x_{2}, x_{3}\right] \cdots\left[x_{d-1}, x_{d}\right]=1, \\
& {\left[r_{0}, x_{2}\right]=\left[\left[x_{1, n} x_{2}\right]\left[x_{2}, x_{3}\right] \cdots\left[x_{d-1}, x_{d}\right], x_{2}\right]=1 \text {, }} \\
& {\left[r_{0, p-1} x_{2}\right]=\left[\left[x_{1, n} x_{2}\right]\left[x_{2}, x_{3}\right] \cdots\left[x_{d-1}, x_{d}\right],{ }_{p-1} x_{2}\right]=1 .}
\end{aligned}
$$


From relations (3.4), one deduces

$$
\left[\left[x_{1, n} x_{2}\right]\left[x_{2}, x_{3}\right]_{,-1} x_{2}\right] \equiv\left[x_{1, n+k-1} x_{2}\right] \cdot\left[x_{3}, k x_{2}\right]^{-1} \equiv 1 \quad \bmod U^{\prime}
$$

for every $1 \leq k \leq p$. Note that one obtains the abelian pro- $p$ group $\bar{U}$ as the quotient of the free $\mathbb{Z}_{p}$-module generated by $\left\{\bar{t}, v_{i, k}\right\}$, with $i=1,3, \ldots, d$ and $0 \leq k \leq p-1$, over the $p$ relations (3.5), as the relations $r_{1}, \ldots, r_{v}$ of $G$ lie in $S^{\prime} \subseteq U^{\prime}$. Moreover, one has $\bar{U} / \bar{U}^{p}=\bar{U} / \Phi(\bar{U}) \simeq U / \Phi(U)$.

Suppose first that $n \geq p$. By applying (3.3) to the $p$ relations (3.5), one obtains $p$ relations in $\bar{U}$,

$$
v_{3, k}=v_{1, n+(k-1)}=\prod_{j=1}^{p-1} v_{1, j}^{\lambda_{j}} \quad \text { for } k=1, \ldots, p,
$$

for some $\lambda_{1}, \ldots, \lambda_{p-1} \in p \mathbb{Z}_{p}$ depending on $k$. Consider the quotient $\bar{U} / \bar{U}^{p}$. The $p$ relations (3.6) translate into

$$
v_{3, k} \equiv 1 \quad \bmod \bar{U}^{p} \quad \text { for } k=1, \ldots, p .
$$

Therefore, the cosets

$$
\left\{\bar{t} \bar{U}^{p}, v_{3,0} \bar{U}^{p}, v_{i, k} \bar{U}^{p}\right\}_{i, k},
$$

with $i=1,4, \ldots, d$ and $0 \leq k<p$, minimally generate the $p$-elementary abelian group $\bar{U} / \bar{U}^{p} \simeq U / \Phi(U)$, and thus $\mathcal{X}_{U}$ minimally generates $U$.

Now suppose that $2<n<p$. By applying (3.3) to the $p$ relations (3.5), one obtains the following $p$ relations in $\bar{U}$ :

$$
\begin{gathered}
v_{1, n+(k-1)}=v_{3, k} \quad \text { for } k=1, \ldots, p-n, \\
v_{3, k}=v_{1, n+(k-1)}=\prod_{j=1}^{p-1} v_{1, j}^{\lambda_{j}} \quad \text { for } k=p-n+1, \ldots, p,
\end{gathered}
$$

for some $\lambda_{1}, \ldots, \lambda_{p-1} \in p \mathbb{Z}_{p}$ depending on $k$. Consider the quotient $\bar{U} / \bar{U}^{p}$. The $p$ relations (3.7) translate into

$$
\begin{aligned}
v_{1, n+(k-1)} & \equiv v_{3, k} \quad \bmod \bar{U}^{p} & & \text { for } k=1, \ldots, p-n, \\
v_{3, k} & \equiv 1 \quad \bmod \bar{U}^{p} & & \text { for } k=p-n+1, \ldots, p .
\end{aligned}
$$

Therefore, the cosets

$$
\left\{\bar{t} \bar{U}^{p}, v_{1, k} \bar{U}^{p}, v_{3, k^{\prime}} \bar{U}^{p}, v_{i, k^{\prime \prime}} \bar{U}^{p}\right\}_{i, k, k^{\prime}, k^{\prime \prime}},
$$

with $i=4, \ldots, d$ and $0 \leq k \leq n-1,0 \leq k^{\prime} \leq p-n, 0 \leq k^{\prime \prime} \leq p-1$, minimally generate the $p$-elementary abelian group $\overline{\bar{U}} / \bar{U}^{p} \simeq U / \Phi(U)$, and thus $\mathcal{X}_{U}$ minimally generates $U$. 
Proposition 3.6. Suppose that $\mathcal{E}=(G, \theta)$ is a Kummerian torsion-free pro-p pair, and let $N$ be the normal subgroup of $U$ generated (as a normal subgroup) by the elements $\left[x_{i}, k x_{2}\right]$, with $i=3, \ldots, d$ and $k \geq 0$. Then one has the following:

(i) $N \subseteq \operatorname{Ker}\left(\left.\theta\right|_{U}\right)$;

(ii) the map $\operatorname{res}_{U, N}^{1}: H^{1}\left(U, \mathbb{F}_{p}\right) \rightarrow H^{1}\left(N, \mathbb{F}_{p}\right)^{U}$ induced by the inclusion $N \subseteq U$ is surjective;

(iii) the pro-p pairs $\left.\mathscr{E}\right|_{U} / N=\left(U / N,\left.\bar{\theta}\right|_{U}\right)$ and $\left.\mathscr{E}\right|_{U}=\left(U,\left.\theta\right|_{U}\right)$ are not Kummerian.

Proof. Since $x_{i} \in \operatorname{Ker}(\theta)$ for every $i=3, \ldots, d$, one has that $N \subseteq \operatorname{Ker}\left(\left.\theta\right|_{U}\right)$. Moreover, $S \subseteq N$.

Let $\mathscr{B}_{U}=\left\{\chi_{t}, \chi_{1, k}, \chi_{3, k^{\prime}}, \chi_{i, k^{\prime \prime}}\right\}_{i, k, k^{\prime}, k^{\prime \prime}}$ denote a basis of $H^{1}\left(U, \mathbb{F}_{p}\right)$ dual to $\chi_{U}$, with $i=4, \ldots, d$ and $0 \leq k \leq l-1,0 \leq k^{\prime} \leq l^{\prime}-1$, and $0 \leq k \leq p-1$, where $l, l^{\prime}$ are as in Lemma 3.5. By duality (2.1), the image of the map

$$
\inf _{U / N, U}^{1}: H^{1}\left(U / N, \mathbb{F}_{p}\right) \rightarrow H^{1}\left(U, \mathbb{F}_{p}\right),
$$

which is injective by (2.2), is the subspace of $H^{1}\left(U, \mathbb{F}_{p}\right)$ generated by the set $\left\{\chi_{t}, \chi_{1, k} \mid 0 \leq k \leq l-1\right\}$. On the other hand, since the elements $\left[x_{i, k} x_{2}\right]$ generate $N$ as a normal subgroup of $U$, one has

$$
\begin{aligned}
\operatorname{dim}\left(H^{1}\left(N, \mathbb{F}_{p}\right)^{U}\right) & =\operatorname{dim}\left(N / N^{p}[U, N]\right) \\
& =\operatorname{dim}\left(H^{1}\left(U, \mathbb{F}_{p}\right)\right)-(1+l) \\
& =\operatorname{dim}\left(H^{1}\left(U, \mathbb{F}_{p}\right)\right)-\operatorname{dim}\left(\operatorname{Im}\left(\inf _{U / N, U}^{1}\right)\right),
\end{aligned}
$$

and therefore the exactness of (2.2) implies that the image of the map $\operatorname{res}_{U, N}^{1}$ is the whole $H^{1}\left(N, \mathbb{F}_{p}\right)^{U}$, i.e., $\operatorname{res}_{U, N}^{1}$ is surjective.

Set $w=\left[x_{1, n-1} x_{2}\right], z=\left[x_{1}, n-1 x_{2}\right]$. Since $\left[z, x_{2}\right]=\left[w, 2 x_{2}\right]=\left[x_{1, n} x_{2}\right] \in N$, by Lemma 3.1, in $U$, one has

$$
\begin{aligned}
{[w, t]=\left[w, x_{2}^{p}\right] } & =\left[w, x_{2}\right]^{p} \cdot\left[w, 2 x_{2}\right]^{\left(\begin{array}{c}
p \\
2
\end{array}\right)} \cdots\left[w, p x_{2}\right] \\
& =z^{p} \cdot\left[z, x_{2}\right]^{\left(\begin{array}{c}
p \\
2
\end{array}\right)} \cdots\left[z, p-1 x_{2}\right] \\
& \equiv z^{p} \quad \bmod N .
\end{aligned}
$$

Since $\chi_{t}, \chi_{1, n-2}, \chi_{1, n-1}$ are linearly independent in $\operatorname{Ker}\left(\operatorname{res}_{U, N}^{1}\right)=\operatorname{Im}\left(\inf _{U / N, U}^{1}\right)$, by duality, the quotient $U / N$ has a minimal generating set containing $t N, w N, z N$ as distinct elements. Therefore, by (3.8) and by [12, Theorem 8.1], the pro- $p$ pair $\left.\mathscr{E}\right|_{U} / N$ is not Kummerian. Finally, by Proposition 2.14, also the pro- $p$ pair $\left.\mathscr{Y}\right|_{U}=\left(U,\left.\theta\right|_{U}\right)$ is not Kummerian. 
Proposition 3.6, together with Theorem 2.8, yields Theorem 1.1 for $r_{0}$ of the first type.

Corollary 3.7. Let $G=\left\langle x_{1}, \ldots, x_{d} \mid r_{0}, r_{1}, \ldots, r_{v}\right\rangle$ be as in Theorem 1.1, with the relation $r_{0}$ of the first type. Then $G$ does not occur as absolute Galois group.

Proof. By Proposition 3.6, $G$ cannot complete into a 1-smooth torsion-free pro- $p$ pair, and thus, by Theorem $2.8, G$ does not occur as maximal pro- $p$ Galois group of a field $\mathbb{K}$ containing a primitive $p$-th root of 1 (and also $\sqrt{-1}$ if $p=2$ ).

Now suppose that $p=2$. It is well known that a field $\mathbb{K}$ contains $\sqrt{-1}$ if and only if, for every epimorphism $\varphi: G_{\mathbb{K}}(2) \rightarrow \mathbb{Z} / 2 \mathbb{Z}$, there exists an epimorphism $\tilde{\varphi}: G_{\mathbb{K}}(2) \rightarrow \mathbb{Z} / 4 \mathbb{Z}$ such that $\varphi=\pi \circ \tilde{\varphi}$, where $\pi: \mathbb{Z} / 4 \mathbb{Z} \rightarrow \mathbb{Z} / 2 \mathbb{Z}$ is the canonical projection (see, e.g., $[35, \S 1.2]$ ). This property holds for $G$, as

$$
G / G^{\prime} \simeq \mathbb{Z} / 2^{f} \mathbb{Z} \times \mathbb{Z}_{2}^{d-1}, \quad \text { with } f \geq 2,
$$

and thus $G$ cannot occur as maximal pro-2 Galois group of a field $\mathbb{K}$ which does not contain $\sqrt{-1}$.

Finally, by Remark 3.3, the pro- $p$ group $G$ does not occur as the absolute Galois group of any field.

\subsection{The second type of $r_{0}$}

In this subsection, $G$ denotes a pro- $p$ group with minimal presentation

$$
G=\left\langle x_{1}, \ldots, x_{d} \mid r_{0}, r_{1} \ldots, r_{v}\right\rangle
$$

as in Theorem 1.1, with $r_{0}$ of the second type, i.e., $d$ is odd and

$$
r_{0}=x_{1}^{q} \cdot\left[x_{1}, n x_{2}\right] \cdot\left[x_{3}, x_{4}\right] \cdots\left[x_{d-1}, x_{d}\right] \cdot s, \quad \text { with } s \in S^{\prime \prime},
$$

where $S$ is the subgroup of $G$ generated by $x_{3}, \ldots, x_{d}$, while $r_{1}, \ldots, r_{v} \in S^{\prime}$. Our goal is to show that, for any orientation $\theta: G \rightarrow 1+p \mathbb{Z}_{p}$, the pair $\mathcal{E}=(G, \theta)$ is not 1-smooth.

First, we establish what a suitable orientation $\theta: G \rightarrow 1+p \mathbb{Z}_{p}$ should be like.

Proposition 3.8. Let $\theta: G \rightarrow 1+p \mathbb{Z}_{p}$ be an orientation of $G$ such that the cyclotomic pro-p pair $\mathcal{G}=(G, \theta)$ is torsion-free. If $\mathcal{G}$ is Kummerian, then $\theta\left(x_{i}\right)=1$ for $i=3, \ldots, d$, and either

(i) $\theta\left(x_{1}\right)=1$ and $\left(\theta\left(x_{2}\right)^{-1}-1\right)^{n}=-q$, or

(ii) $\theta\left(x_{1}\right) \neq 1$ and $\theta\left(x_{2}\right)=1$ (this is possible only if $q=0$ ).

In particular, if $-q \notin\left(1+p \mathbb{Z}_{p}\right)^{n}$, then $\mathcal{G}$ is not Kummerian for any orientation $\theta: G \rightarrow 1+p \mathbb{Z}_{p}$ 
Proof. First, note that $\left.\theta\right|_{S^{\prime}} \equiv \mathbf{1}$, and thus, by Lemma 3.2, one has $\left.c\right|_{S^{\prime \prime}} \equiv \mathbf{0}$ for every continuous 1 -cocycle $c: G \rightarrow \mathbb{Z}_{p}(\theta)$. From

$$
1=\theta(1)=\theta\left(x_{1}^{q}\left[x_{1, n} x_{2}\right]\left[x_{3}, x_{4}\right] \cdots\left[x_{d-1}, x_{d}\right] \cdot s\right)=\theta\left(x_{1}\right)^{q},
$$

we deduce $\theta\left(x_{1}\right)^{q}=1$. Since $\operatorname{Im}(\theta)$ is torsion-free, if $q \neq 0$, one has $\theta\left(x_{1}\right)=1$, while one might have $\theta\left(x_{1}\right) \neq 1$ only if $q=0$.

For every $i=1, \ldots, d$, let $c_{i}: G \rightarrow \mathbb{Z}_{p}(\theta)$ be the continuous 1-cocycle such that $c_{i}\left(x_{j}\right)=\delta_{i j}$, with $j \in\{1, \ldots, d\}$ (such a continuous 1-cocycle always exists by Proposition 2.16, since we are assuming that $\mathcal{E}$ is Kummerian). Then

$$
\begin{aligned}
0 & =c_{i}\left(x_{1}^{q}\left[x_{1, n} x_{2}\right]\left[x_{3}, x_{4}\right] \cdots\left[x_{d-1}, x_{d}\right]\right) \\
& =c_{i}\left(x_{1}^{q}\right)+\theta\left(x_{1}\right)^{q} \cdot c_{i}\left(\left[x_{1, n} x_{2}\right]\left[x_{3}, x_{4}\right] \cdots\left[x_{d-1}, x_{d}\right]\right) \\
& =c_{i}\left(x_{1}^{q}\right)+c_{i}\left(\left[x_{1, n} x_{2}\right]\right)+c_{i}\left(\left[x_{3}, x_{4}\right]\right)+\cdots+c_{i}\left(\left[x_{d-1}, x_{d}\right]\right) .
\end{aligned}
$$

For $i \geq 3, i$ odd, from (2.7), Lemma 3.2, and (3.9), we get

$$
0=0+\cdots+c_{i}\left(\left[x_{i}, x_{i+1}\right]\right)+\cdots+0=\theta\left(x_{i} x_{i+1}\right)^{-1}\left(1-\theta\left(x_{i+1}\right)\right) \cdot 1,
$$

and hence $\theta\left(x_{i+1}\right)=1$. Analogously, for $i \geq 4, i$ even, one gets

$$
0=0+\cdots+c_{i}\left(\left[x_{i-1}, x_{i}\right]\right)+\cdots+0=\theta\left(x_{i-1} x_{i}\right)^{-1}\left(-1+\theta\left(x_{i-1}\right)\right) \cdot 1,
$$

and hence $\theta\left(x_{i-1}\right)=1$. On the other hand, for $i=1$, from (2.7), Lemma 3.2, and (3.9), we get

$0=c_{1}\left(x_{1}^{q}\right)+c_{1}\left(\left[x_{1, n} x_{2}\right]\right)+0= \begin{cases}q+\left(\theta\left(x_{2}\right)^{-1}-1\right)^{n} & \text { if } \theta\left(x_{1}\right)=1, \\ 0+\theta\left(x_{1}\right)^{-1}\left(\theta\left(x_{2}\right)^{-1}-1\right)^{n} & \text { if } \theta\left(x_{1}\right) \neq 1\end{cases}$

(recall that in the second case necessarily $q=0$ ): in the first case, one deduces (i); in the second case, one deduces (ii).

Note that if $\mathcal{E}=(G, \theta)$ is a Kummerian pro- $p$ pair, then $S \subseteq \operatorname{Ker}(\theta)$, and thus Lemma 3.2 implies that $\left.c\right|_{S^{\prime}} \equiv \mathbf{0}$ for every continuous 1-cocycle $c: G \rightarrow \mathbb{Z}_{p}(\theta)$. From now on, we assume that $-q=\lambda^{n}$ for some $\lambda \in p \mathbb{Z}_{p}$ so that $G$ may be completed into a Kummerian pro- $p$ pair $\mathscr{E}=(G, \theta)$.

Let $\phi: G \rightarrow \mathbb{Z} / p \mathbb{Z}$ be the homomorphism defined by

$$
x_{i} \mapsto 0 \quad \text { for } i=1,3,4, \ldots, d \quad \text { and } \quad x_{2} \mapsto 1,
$$

and set $U=\operatorname{Ker}(\phi)$. 
Lemma 3.9. The set

$$
X_{U}=\left\{\begin{array}{c}
t:=x_{2}^{p}, \\
x_{1},\left[x_{1}, x_{2}\right], \ldots,\left[x_{1, m-1} x_{2}\right], \\
x_{3},\left[x_{3}, x_{2}\right], \ldots,\left[x_{3}, p-1 x_{2}\right], \\
\vdots \\
x_{d},\left[x_{d}, x_{2}\right], \ldots,\left[x_{d, p-1} x_{2}\right]
\end{array}\right\},
$$

where $m=\min \{n, p\}$, is a minimal generating set of $U$.

Proof. Set $\bar{U}=U / U^{\prime}$, and for $g \in U$, let $\bar{g}=g \cdot U^{\prime}$ denote its image in $\bar{U}$. In particular, we set $v_{i, 0}=\bar{x}_{i}$ and $v_{i, k}=\overline{\left[x_{i}, k_{2} x_{2}\right]} \in \bar{U}$ for $i=1, \ldots, d$ and $k \geq 1$.

The group $U$ is generated as normal subgroup of $G$ by $t$ and $x_{1}, x_{3}, x_{4}, \ldots, x_{d}$. Since $G^{\prime} \subseteq U$, one has $G^{\prime \prime} \subseteq U^{\prime}$. Also, $S^{\prime} \subseteq U^{\prime}$, as $S \subseteq U$. By Lemma 3.1, for every $i=1,3, \ldots, d$, one has

$$
\left[x_{i}, t\right] \equiv\left(\prod_{k=1}^{p-1}\left[x_{i}, x_{2} x_{2}\right]^{\left(\begin{array}{c}
p \\
k
\end{array}\right)}\right) \cdot\left[x_{i}, p x_{2}\right] \bmod U^{\prime},
$$

and since $\left[x_{i}, t\right] \in U^{\prime}$, it follows that, for every $k \geq p$,

$$
v_{i, k}=v_{i, 1}^{\lambda_{1}} \cdot v_{i, 2}^{\lambda_{2}} \cdots v_{i, p-2}^{\lambda_{p-2}} \cdot v_{i, p-1}^{\lambda_{p-1}}
$$

for some $\lambda_{1}, \ldots, \lambda_{p-1} \in p \mathbb{Z}_{p}$. Hence, $\bar{U}$ is generated as an abelian pro- $p$ group by the set $\left\{\bar{t}, v_{i, k}\right\}_{i, k}$, with $i=1,3, \ldots, d$ and $0 \leq k \leq p-1$.

In $U$, from the defining relation $r_{0}=1$, one obtains $p$ relations

$$
\begin{array}{r}
r_{0}=x_{1}^{q}\left[x_{1}, n x_{2}\right]\left[x_{3}, x_{4}\right] \cdots\left[x_{d-1}, x_{d}\right]=1, \\
{\left[r_{0}, x_{2}\right]=\left[x_{1}^{q}\left[x_{1}, n x_{2}\right]\left[x_{3}, x_{4}\right] \cdots\left[x_{d-1}, x_{d}\right], x_{2}\right]=1,} \\
\vdots \\
{\left[r_{0, p-1} x_{2}\right]=\left[x_{1}^{q}\left[x_{1}, n x_{2}\right]\left[x_{3}, x_{4}\right] \cdots\left[x_{d-1}, x_{d}\right], p-1 x_{2}\right]=1 .}
\end{array}
$$

From relations (3.11), one deduces

$$
\left[x_{1}^{q} \cdot\left[x_{1, n} x_{2}\right]_{, k} x_{2}\right] \equiv\left[x_{1}, k x_{2}\right]^{q} \cdot\left[x_{1}, n+k x_{2}\right] \equiv 1 \quad \bmod U^{\prime}
$$

for every $0 \leq k \leq p-1$. Note that one obtains the abelian pro- $p$ group $\bar{U}$ as the quotient of the $\mathbb{Z}_{p}$-module $A$ - where $A$ is the free $\mathbb{Z}_{p}$-module generated by $\left\{\bar{t}, v_{i, k}\right\}$, with $i=1,3, \ldots, d$ and $0 \leq k \leq p-1-$ over the $p$ relations (3.12), as 
the relations $r_{1}, \ldots, r_{v}$ of $G$ lie in $S^{\prime} \subseteq U^{\prime}$, and this yields an epimorphism of abelian pro- $p$ groups $\pi: A \rightarrow \bar{U}$. Moreover, one has

$$
\bar{U} / \bar{U}^{p}=\bar{U} / \Phi(\bar{U}) \simeq U / \Phi(U) .
$$

Suppose first that $n \geq p$. By applying (3.10) to the $p$ relations (3.12), one obtains $p$ relations in $\bar{U}$,

$$
v_{1, k}^{-q}=v_{1, n+k}=\prod_{j=1}^{p-1} v_{1, j}^{\lambda_{j}} \quad \text { for } k=0, \ldots, p-1,
$$

for some $\lambda_{1}, \ldots, \lambda_{p-1} \in p \mathbb{Z}_{p}$ depending on $k$. Since $p \mid q$, one has $\operatorname{Ker}(\pi) \subseteq p A$ (here we use the additive notation for $A$ ). Therefore, the set $\left\{\bar{t}, v_{i, k}\right\}_{i, k}$, with $i=1,3, \ldots, d$ and $0 \leq k<p$, is a minimal generating set of $\bar{U}$, and the isomorphism $U / \Phi(U) \simeq \bar{U} / \Phi(\bar{U})$ implies that $\mathcal{X}_{U}$ minimally generates $U$.

Suppose now that $2<n<p$. By applying (3.10) to the $p$ relations (3.12), one obtains the following $p$ relations in $\bar{U}$ :

$$
\begin{aligned}
v_{1, n+k} & =v_{1, k}^{-q} \quad \text { for } k=0, \ldots, p-n-1, \\
v_{1, k}^{-q}=v_{1, n+k} & =\prod_{j=1}^{p-1} v_{1, j}^{\lambda_{j}} \quad \text { for } k=p-n, \ldots, p-1,
\end{aligned}
$$

for some $\lambda_{1}, \ldots, \lambda_{p-1} \in p \mathbb{Z}_{p}$ depending on $k$. Consider the quotient

$$
\bar{U} / \bar{U}^{p}=\bar{U} / \Phi(\bar{U})
$$

which is isomorphic to $U / \Phi(U)$. The $p-n$ relations (3.13) translate into

$$
v_{1, n+k} \equiv 1 \quad \bmod \bar{U}^{p} \quad \text { for } k=0, \leq, p-n-1,
$$

while the $n$ relations (3.13) become trivial in $\bar{U} / \bar{U}^{p}$. Therefore, the cosets

$$
\left\{\bar{t} \bar{U}^{p}, v_{1, k} \bar{U}^{p}, v_{i, k^{\prime}} \bar{U}^{p} \mid i=3, \ldots, d ; 0 \leq k \leq n-1 ; 0 \leq k^{\prime} \leq p-1\right\}
$$

minimally generate the $p$-elementary abelian pro- $p$ group $\bar{U} / \bar{U}^{p} \simeq U / \Phi(U)$, and thus $\mathcal{X}_{U}$ minimally generates $U$.

Remark 3.10. Suppose that the pro- $p$ pair $\mathscr{G}=(G, \theta)$ is Kummerian. Since

$$
G^{\prime} \subseteq \operatorname{Ker}\left(\left.\theta\right|_{U}\right),
$$


one has $G^{\prime \prime} \subseteq \operatorname{Ker}\left(\left.\theta\right|_{U}\right)^{\prime}$. If $x_{1} \in \operatorname{Ker}\left(\left.\theta\right|_{U}\right)$, then for every $m \geq 1$, one has

$$
\begin{aligned}
{\left[x_{1}^{q}, m x_{2}\right] } & =\left[\left[x_{1}, x_{2}\right]^{x_{1}^{q-1}} \cdots\left[x_{1}, x_{2}\right]^{x_{1}} \cdot\left[x_{1}, x_{2}\right], m-1\right. \\
& \equiv\left[\left[x_{1}, x_{2}\right]^{q},{ }_{m-1} x_{2}\right] \bmod \operatorname{Ker}\left(\left.\theta\right|_{U}\right)^{\prime} \\
& \equiv\left[x_{1}, m x_{2}\right]^{q} \bmod \operatorname{Ker}\left(\left.\theta\right|_{U}\right)^{\prime},
\end{aligned}
$$

as $y^{x_{1}} \equiv y \bmod \operatorname{Ker}\left(\left.\theta\right|_{U}\right)^{\prime}$ for every $y \in \operatorname{Ker}\left(\left.\theta\right|_{U}\right)$. On the other hand, if one has $x_{1} \notin \operatorname{Ker}\left(\left.\theta\right|_{U}\right)$, then $q=0$ by Proposition 3.8, and (3.14) holds trivially.

Proposition 3.11. Suppose that $\mathcal{E}=(G, \theta)$ is a Kummerian torsion-free pro- $p$ pair, and set $N=\operatorname{Ker}\left(\left.\theta\right|_{U}\right)^{\prime}$. Then the pro-p pairs $\left.\mathscr{Y}\right|_{U} / N=\left(U / N,\left.\bar{\theta}\right|_{U}\right)$ and $\left.\mathcal{E}\right|_{U}=\left(U,\left.\theta\right|_{U}\right)$ are not Kummerian.

Proof. Recall that $G^{\prime \prime} \subseteq N, S^{\prime} \subseteq N$, and the commutators $\left[x_{3}, x_{4}\right], \ldots,\left[x_{d-1}, x_{d}\right]$ lie in $N$. Moreover, one has $p^{n} \mid q$, by Proposition 3.8.

Set $w=\left[x_{1, n-2} x_{2}\right], z=\left[x_{1}, n-1 x_{2}\right]=\left[w, x_{2}\right]$. Then, by Lemma 3.9, $t, w, z$ are distinct elements of the minimal generating set $x_{U} \subseteq U$. Since

$$
\left[z, x_{1}\right] \equiv x_{1}^{-q} \bmod N \text {, }
$$

by Lemma 3.1 and by Remark 3.10, one has

$$
\begin{aligned}
& {[w, t] \equiv\left[w, x_{2}\right]^{p}\left[w, 2 x_{2}\right]^{\left(\begin{array}{c}
p \\
2
\end{array}\right)} \cdots\left[w, p-1 x_{2}\right]^{p}\left[w, p x_{2}\right] \bmod N} \\
& \equiv z^{p}\left[z, x_{2}\right]^{\left(\begin{array}{l}
p \\
2
\end{array}\right)}\left[z, 2 x_{2}\right]^{\left(\begin{array}{c}
p \\
3
\end{array}\right)} \cdots\left[z, p-2 x_{2}\right]^{p}\left[z, p-1 x_{2}\right] \bmod N \\
& \equiv z^{p} \cdot x_{1}^{-q\left(\begin{array}{c}
p \\
2
\end{array}\right)} \cdot\left[x_{1}^{-q}, x_{2}\right]^{\left(\begin{array}{c}
p \\
3
\end{array}\right)} \ldots \\
& \cdot\left[x_{1}^{-q}, p-3 x_{2}\right]^{p}\left[x_{1}^{-q}, p-2 x_{2}\right] \bmod N \\
& \equiv z^{p} \cdot x_{1}^{-q\left(\begin{array}{l}
p \\
2
\end{array}\right)}\left[x_{1}, x_{2}\right]^{-q\left(\begin{array}{c}
p \\
3
\end{array}\right)} \ldots \\
& \cdot\left[x_{1}, p-3 x_{2}\right]^{-q p}\left[x_{1}, p-2 x_{2}\right]^{-q} \bmod N \text {. }
\end{aligned}
$$

Now we have three cases, depending on the value of $n$.

(a) If $n \geq p$, then from (3.15), one has

$$
z^{p} \equiv[w, t] \cdot x_{1}^{q\left(\begin{array}{c}
p \\
2
\end{array}\right)}\left[x_{1}, x_{2}\right]^{q\left(\begin{array}{c}
p \\
3
\end{array}\right)} \cdots\left[x_{1}, p-3 x_{2}\right]^{q p} w^{q} \bmod N .
$$

(b) If $n=p-1$, then from (3.15), one has

$$
[w, t] \equiv z^{p} \cdot x_{1}^{-q\left(\begin{array}{c}
p \\
2
\end{array}\right)} \cdot\left[x_{1}, x_{2}\right]^{-q\left(\begin{array}{c}
p \\
3
\end{array}\right)} \cdots w^{-q p} \cdot z^{-q} \bmod N
$$


and thus

$$
z^{p-q} \equiv[w, t] \cdot x_{1}^{q\left(\begin{array}{c}
p \\
2
\end{array}\right)} \cdot\left[x_{1}, x_{2}\right]^{q\left(\begin{array}{c}
p \\
3
\end{array}\right)} \cdots\left[x_{1, n-3} x_{2}\right]^{q\left(\begin{array}{c}
p \\
p-2
\end{array}\right)} \cdot w^{q p} \bmod N .
$$

Since $p^{n} \mid q$, one has $p-q \neq 0$.

(c) If $n<p-1$, after iterating the replacement $\left[x_{1}, k x_{2}\right] \equiv\left[x_{1, k-n} x_{2}\right]^{-q} \bmod N$ in the last line of (3.15) whenever $k \geq n$, one obtains

$$
z^{p+\lambda_{n-1}} \equiv[w, t] \cdot x_{1}^{\lambda_{0}} \cdot\left[x_{1}, x_{2}\right]^{\lambda_{1}} \cdots w^{\lambda_{n-1}} \bmod N,
$$

with $\lambda_{0}, \ldots, \lambda_{n-1} \in p^{2} \mathbb{Z}_{p}$, as $p^{2} \mid q$. In particular, $p+\lambda_{n-1} \neq 0$.

In all three cases, [12, Theorem 8.1] implies that the pro- $p$ pair $\left.\mathscr{E}\right|_{U} / N$ cannot be Kummerian.

Finally, as $N \subseteq K\left(\left.\mathscr{G}\right|_{U}\right)$, by Theorem 2.10, also the pro- $p$ pair $\left.\mathscr{G}\right|_{U}=\left(U,\left.\theta\right|_{U}\right)$ cannot be Kummerian.

Proposition 3.11, together with Theorem 2.8, yields Theorem 1.1 for $r_{0}$ of the second type.

Corollary 3.12. Let $G=\left\langle x_{1}, \ldots, x_{d} \mid r_{0}, r_{1}, \ldots, r_{v}\right\rangle$ be as in Theorem 1.1, with the relation $r_{0}$ of the second type. Then $G$ does not occur as absolute Galois group.

Proof. The proof is verbatim the same as the proof of Corollary 3.7.

\section{One-relator pro- $p$ groups that are not absolute Galois groups}

In the next two sections, we focus on those pro- $p$ groups defined in Theorem 1.1 with a single defining relation - i.e., $v=0$.

\subsection{Quadratic cohomology}

Let $G$ be a one-relator pro- $p$ group of one of the two families defined in Theorem 1.1. Then one has either

$$
\begin{aligned}
& r_{0} \equiv\left[x_{2}, x_{3}\right] \cdots\left[x_{d-1}, x_{d}\right] \quad \bmod G_{(3)} \quad \text { or } \\
& r_{0} \equiv\left[x_{3}, x_{4}\right] \cdots\left[x_{d-1}, x_{d}\right] \bmod G_{(3)} \text {. }
\end{aligned}
$$

Hence, the $\mathbb{F}_{p}$-cohomology algebra $H^{\bullet}\left(G, \mathbb{F}_{p}\right)$ of $G$ is quadratic by Proposition 2.1. In particular, let $\mathscr{B}=\left\{\chi_{1}, \ldots, \chi_{d}\right\}$ be a basis of $H^{1}\left(G, \mathbb{F}_{p}\right)$ dual to $\mathcal{X}=\left\{x_{1}, \ldots, x_{d}\right\}$. Then one has the following. 
(a) If $r_{0}$ is of the first type, then

$$
\begin{aligned}
& \chi_{2} \cup \chi_{3}=\cdots=\chi_{d-1} \cup \chi_{d} \neq 0, \\
& \chi_{i} \cup \chi_{j} \text { for } 1 \leq i<j \leq d,(i, j) \neq(2,3), \ldots,(d-1, d),
\end{aligned}
$$

and $\chi_{2} \cup \chi_{3}$ generates $H^{2}\left(G, \mathbb{F}_{p}\right)$. In particular,

$$
\begin{aligned}
H^{1}\left(G, \mathbb{F}_{p}\right)^{\perp} & =\left\{\chi \in H^{1}\left(G, \mathbb{F}_{p}\right) \mid \chi \cup \psi=0 \text { for all } \psi \in H^{1}\left(G, \mathbb{F}_{p}\right)\right\} \\
& =\operatorname{Span}_{\mathbb{F}_{p}}\left(\chi_{1}\right) .
\end{aligned}
$$

(b) If $r_{0}$ is of the second type, then

$$
\begin{aligned}
& \chi_{3} \cup \chi_{4}=\cdots=\chi_{d-1} \cup \chi_{d} \neq 0, \\
& \chi_{i} \cup \chi_{j} \quad \text { for } 1 \leq i<j \leq d,(i, j) \neq(3,4), \ldots,(d-1, d),
\end{aligned}
$$

and $\chi_{3} \cup \chi_{4}$ generates $H^{2}\left(G, \mathbb{F}_{p}\right)$. In particular,

$$
\begin{aligned}
H^{1}\left(G, \mathbb{F}_{p}\right)^{\perp} & =\left\{\chi \in H^{1}\left(G, \mathbb{F}_{p}\right) \mid \chi \cup \psi=0 \text { for all } \psi \in H^{1}\left(G, \mathbb{F}_{p}\right)\right\} \\
& =\operatorname{Span}_{\mathbb{F}_{p}}\left(\chi_{1}, \chi_{2}\right) .
\end{aligned}
$$

This proves Proposition 1.2(i).

Remark 4.1. Since $H^{k}\left(G, \mathbb{F}_{p}\right)=0$ for $k \geq 3$, the pro- $p$ group $G$ is torsion-free (cf., e.g., [32, Proposition A]).

\subsection{Free and abelian subgroups}

Recall from Proposition 2.6 that Bloch-Kato pro- $p$ groups contain a unique maximal normal abelian subgroups, and that if a Bloch-Kato pro- $p$ group is not metaabelian, then it contains a free non-abelian subgroup. We prove that the one-relator pro- $p$ groups defined in Theorem 1.2 satisfy these two group-theoretic properties.

Proposition 4.2. Set $G=\left\langle x_{1}, \ldots, x_{d} \mid r_{0}\right\rangle$ as in Theorem 1.2. Then

(i) G contains a free non-abelian subgroup;

(ii) G contains no normal abelian subgroups.

Proof. By [30, Proposition 4.10], one has a short exact sequence of pro- $p$ groups

$$
\{1\} \rightarrow N \rightarrow G \rightarrow G / N \rightarrow\{1\}
$$


where $N$ is the normal subgroup of $G$ generated by $x_{1}$, respectively by $x_{1}, x_{2}$, depending on the family of $G$, such that $N$ is a free non-abelian pro- $p$ group, and $G / N$ is a torsion-free Demushkin group on $d-1$, respectively $d-2$, generators. This proves (i).

Suppose that $A$ is a non-trivial normal abelian subgroup of $G$. Since $G$ is torsion-free by Remark 4.1, $A$ is a free abelian pro- $p$ group, i.e., $A$ is isomorphic to the direct product of (possibly infinite) copies of $\mathbb{Z}_{p}$. Since $N$ is free and non-abelian, one has $A \cap N=\{1\}$, and thus

$$
A=A /(A \cap N) \simeq A N / N \unlhd G / N .
$$

It is well known that a torsion-free Demushkin group has a non-trivial normal abelian subgroup if and only if it has 2 generators. Thus,

$$
G / N \simeq\left\langle\bar{x}_{i}, \bar{x}_{i+1} \mid\left[\bar{x}_{i}, \bar{x}_{i+1}\right]=1\right\rangle \simeq \mathbb{Z}_{p}^{2},
$$

where $i=2$, respectively $i=3$, and $\bar{x}_{j}=x_{j} N$ denotes the image of $x_{j}$ in $G / N$ for $j=i, i+1$. In particular, $A \simeq \mathbb{Z}_{p}^{a}$, with $a \in\{1,2\}$, since $A \unlhd G / N$ by (4.3).

Since $G / N$ is abelian and $A$ is normal in $G$, one has

$$
N \supseteq G^{\prime} \supseteq[G, A] \subseteq A,
$$

and since $A \cap N=\{1\}$, one deduces $[G, A]=\{1\}$, i.e., $A$ is contained in the center $\mathrm{Z}(G)$ of $G$. Hence, for every $x \in G \backslash A$, the subgroup $\langle x\rangle A$ of $G$ is abelian and torsion-free so that $\langle x\rangle A \simeq \mathbb{Z}_{p}^{a+1}$. In particular, if $x^{p^{k}} \in A$ for some $x \in G$ and $k \geq 1$, then $x \in A$, and consequently, $G / A$ is a torsion-free pro- $p$ group. Since the $\mathbb{F}_{p}$-cohomology algebra $H^{\bullet}\left(G, \mathbb{F}_{p}\right)$ is quadratic, from [33, Proposition 7.11], one deduces that $A \nsubseteq \Phi(G)$, and $G \simeq A \times(G / A)$. Then, by [25, Theorem 2.4.6], one has

$$
\begin{aligned}
H^{2}\left(G, \mathbb{F}_{p}\right) & \simeq \bigoplus_{s+t=2} H^{s}\left(G / A, H^{t}\left(A, \mathbb{F}_{p}\right)\right) \\
& \simeq H^{2}\left(G / A, \mathbb{F}_{p}\right) \oplus H^{1}\left(G / A, \mathbb{F}_{p}\right) \oplus H^{2}\left(A, \mathbb{F}_{p}\right),
\end{aligned}
$$

as $G / A$ acts trivially on $H^{t}\left(A, \mathbb{F}_{p}\right), t=1,2$, and this contradicts (4.1)-(4.2). Therefore, $A=\{1\}$, and this proves (ii).

Therefore, one may not employ Proposition 2.6 to show that the pro- $p$ groups of the two families defined in Theorem 1.1 are not Bloch-Kato - and thus that they cannot occur as absolute Galois groups of fields. We ask the following.

Question 4.3. Are (some of) the one-relator pro- $p$ groups defined in Theorem 1.2 Bloch-Kato pro- $p$ groups? 


\subsection{Benson-Lemire-Minac-Swallow's examples}

For $x, y$ elements of a (pro- $p$ ) groups $G$ and $n \geq 1$, set

$$
\left.\left.\left[x_{n}, y\right]=[\underbrace{x, \ldots,[x[x}_{n \text { times }}, y]\right] \ldots\right] .
$$

Let $p$ be odd, and let $G$ be a finitely generated one-relator pro- $p$ group $G$ with minimal presentation $G=\left\langle y_{1}, \ldots, y_{d} \mid t\right\rangle, d \geq 4$, and defining relation

$$
t=y_{1}^{q} \cdot\left[y_{1 n}, y_{2}\right]\left[y_{3}, y_{4}\right] \cdots\left[y_{m-1}, y_{m}\right] \cdot\left[y_{1}^{p}, y_{k_{1}}\right] \cdots\left[y_{1}^{p}, y_{k_{v}}\right],
$$

where

(a) $q=p^{f}$ with $f \in\{1,2, \ldots, \infty\}$ and $2 \leq n \leq p-1$,

(b) $4 \leq m \leq d$ is even,

(c) $v \geq 0$ and $3 \leq k_{1}<\cdots<k_{v} \leq d$ if $v \neq 0$,

(d) $\{3, \ldots, m\} \cup\left\{k_{1}, \ldots, k_{v}\right\}=\{3, \ldots, d\}$.

This family of one-relator pro- $p$ groups was introduced by Benson et al. in [2] for short, we shall call these pro- $p$ groups BLMS pro- $p$ groups. In [2, Corollary 1], it is shown that BLMS pro- $p$ groups do not occur as maximal pro- $p$ Galois groups of fields containing a primitive $p$-th root of 1 . We show that the intersection of the family of BLMS pro- $p$ groups with the family of one-relator pro- $p$ groups defined in Theorem 1.2 is rather small.

Proposition 4.4. Let $G=\left\langle x_{1}, \ldots, x_{d} \mid r_{0}\right\rangle$ be a one-relator pro- $p$ group as defined in Theorem 1.2, and suppose that $G$ may complete into a Kummerian pro- $p$ pair $\mathcal{E}=(G, \theta)$. Then $G$ is a BLMS pro-p group only if

$$
r_{0}=\left[x_{1}, n x_{2}\right]\left[x_{3}, x_{4}\right] \cdots\left[x_{d-1}, x_{d}\right] \cdot s .
$$

Proof. Suppose that $G$ is a BLMS pro- $p$ group. Thus, $G=\left\langle y_{1}, \ldots, y_{d} \mid t\right\rangle$ with $t$ as above. Since

$$
t \equiv\left[y_{3}, y_{4}\right] \cdots\left[y_{m-1}, y_{m}\right] \bmod G_{(3)},
$$

by Proposition 2.1 , the $\mathbb{F}_{p}$-cohomology algebra $H^{\bullet}\left(G, \mathbb{F}_{p}\right)$ is quadratic, and in particular, one has $\operatorname{dim}\left(H^{1}\left(G, \mathbb{F}_{p}\right)^{\perp}\right)=2+d-m$. Hence, by (4.1)-(4.2), one has that $\operatorname{dim}\left(H^{1}\left(G, \mathbb{F}_{p}\right)^{\perp}\right)=2$ so that $r_{0}$ is necessarily of the second type, i.e.,

$$
r_{0}=x_{1}^{q}\left[x_{1, n} x_{2}\right]\left[x_{3}, x_{4}\right] \cdots\left[x_{d-1}, x_{d}\right] \cdot s
$$

for some $s \in S^{\prime \prime}$, where $S$ is the subgroup of $G$ generated by $x_{1}, x_{3}, \ldots, x_{d}$. 
We claim that, in (4.5), one has $q=0$ so that $r_{0}$ is as stated in (4.4). For this purpose, let $\theta: G \rightarrow 1+p \mathbb{Z}_{p}$ be an orientation such that the pro- $p$ pair $\mathscr{E}=(G, \theta)$ is Kummerian. Then $\theta\left(y_{1}\right)^{q}=\theta(t)=1$, and moreover, Proposition 2.16 yields $c(t)=0$ for every continuous 1 -cocycle $c: G \rightarrow \mathbb{Z}_{p}(\theta)$. For every $i=1, \ldots, d$, let $c_{i}: G \rightarrow \mathbb{Z}_{p}(\theta)$ be the continuous 1-cocycle such that $c_{i}\left(y_{j}\right)=\delta_{i j}$. Then one has

$$
\begin{aligned}
0= & c_{i}\left(y_{1}^{q}\left[y_{1 n}, y_{2}\right]\left[y_{3}, y_{4}\right] \cdots\left[y_{d-1}, y_{d}\right] \cdot\left[y_{1}^{p}, y_{k_{1}}\right] \cdots\left[y_{1}^{p}, y_{k_{v}}\right]\right) \\
= & c_{i}\left(y_{1}^{p}\right)+c_{i}\left(\left[y_{1 n}, y_{2}\right]\right)+c_{i}\left(\left[y_{3}, y_{4}\right]\right)+\cdots+c_{i}\left(\left[y_{d-1}, y_{d}\right]\right) \\
& \quad+\sum_{j=1}^{v} c_{i}\left(\left[y_{1}^{p}, y_{k_{j}}\right]\right) .
\end{aligned}
$$

By Lemma 3.2, from (4.6), one obtains

$$
0=c_{2}(t)=c_{2}\left(\left[y_{1 n}, y_{2}\right]\right)=\left(1-\theta\left(y_{1}\right)^{-1}\right)^{n},
$$

and thus $\theta\left(y_{1}\right)=1$. For $i \geq 3, i$ odd, by Lemma 3.2, from (4.6), one obtains

$$
0=\left\{\begin{array}{l}
\theta\left(y_{i} y_{i+1}\right)^{-1}\left(1-\theta\left(y_{i+1}\right)\right) \\
\quad \text { if } i \neq k_{j} \text { for all } j=1, \ldots, v, \\
\theta\left(y_{i} y_{i+1}\right)^{-1}\left(1-\theta\left(y_{i+1}\right)\right)+\theta\left(y_{i}\right)^{-1}\left(1-\theta\left(y_{1}\right)^{p}\right) \\
\quad \text { if } i \in\left\{k_{1}, \ldots, k_{v}\right\},
\end{array}\right.
$$

and in both cases, one has $\theta\left(y_{i+1}\right)=1$, as $\theta\left(y_{1}\right)=1$. Analogously, one obtains $\theta\left(y_{i-1}\right)=1$ for $i \geq 3, i$ even. Finally,

$$
0=c_{1}\left(x_{1}^{q}\right)+\sum_{j=1}^{v} c_{1}\left(\left[y_{1}^{p}, y_{k_{j}}\right]\right)=q+\sum_{j=1}^{v}\left(\theta\left(y_{k_{j}}\right)^{-1}-1\right) \cdot p=q+0,
$$

and hence $q=0$.

Therefore, most of the one-relator pro- $p$ groups defined in Theorem 1.2 are not BLMS pro- $p$ groups.

\section{Massey products}

\subsection{Massey products and pro- $p$ groups}

Let $G$ be a pro- $p$ group. For $m \geq 2$, the $m$-fold Massey product on $H^{1}\left(G, \mathbb{F}_{p}\right)$ is a multi-valued map

$$
\underbrace{H^{1}\left(G, \mathbb{F}_{p}\right) \times \ldots \times H^{1}\left(G, \mathbb{F}_{p}\right)}_{m \text { times }} \rightarrow H^{2}\left(G, \mathbb{F}_{p}\right) .
$$


For $m \geq 2$ elements $\psi_{1}, \ldots, \psi_{m}$ of $H^{1}\left(G, \mathbb{F}_{p}\right)$, we write $\left\langle\psi_{1}, \ldots, \psi_{m}\right\rangle$ for the set of values of the $m$-fold Massey product of the elements $\psi_{1}, \ldots, \psi_{m}$. If $m=2$, then the 2-fold Massey product coincides with the cup-product, i.e.,

$$
\left\langle\psi_{1}, \psi_{2}\right\rangle=\left\{\psi_{1} \cup \psi_{2}\right\} \subseteq H^{2}\left(G, \mathbb{F}_{p}\right) \text { for } \psi_{1}, \psi_{2} \in H^{1}\left(G, \mathbb{F}_{p}\right)
$$

For further details on this operation in the general homological context, we direct the reader to $[6,19]$, and to $[9,24,38]$ for Massey products in the profinite and Galois-theoretic context. In particular, the definition of $m$-fold Massey products in the $\mathbb{F}_{p}$-cohomology of pro- $p$ groups may be found in [24, Definition 2.1]. For the purposes of our investigation, the group-theoretic conditions given by Proposition 5.3 below will be enough.

Given $m \geq 2$ elements

$$
\psi_{1}, \ldots, \psi_{m} \in H^{1}\left(G, \mathbb{F}_{p}\right),
$$

the Massey product $\left\langle\psi_{1}, \ldots, \psi_{m}\right\rangle$ is said to be defined if it is non-empty, and it is said to vanish if it contains 0 . In the following proposition, we collect some properties of Massey products (cf., e.g., [38, § 1.2] and [24, § 2]).

Proposition 5.1. Let $G$ be a pro- $p$ group and $\psi_{1}, \ldots, \psi_{m} \in H^{1}\left(G, \mathbb{F}_{p}\right), m \geq 3$.

(i) If $\psi_{k}=0$ for some $k \in\{1, \ldots, m\}$ and the Massey product $\left\langle\psi_{1}, \ldots, \psi_{m}\right\rangle$ is defined, then $0 \in\left\langle\psi_{1}, \ldots, \psi_{m}\right\rangle$.

(ii) If the Massey product $\left\langle\psi_{1}, \ldots, \psi_{m}\right\rangle$ is defined, then $\psi_{i} \cup \psi_{i+1}=0$ for all $i=1, \ldots, m-1$.

(iii) If the Massey product $\left\langle\psi_{1}, \ldots, \psi_{m}\right\rangle$ is defined, then one has the inclusion

$$
\left\langle\psi_{1}, \ldots, \psi_{m}\right\rangle \supseteq \xi+\psi_{1} \cup H^{1}\left(G, \mathbb{F}_{p}\right)+\psi_{m} \cup H^{1}\left(G, \mathbb{F}_{p}\right),
$$

where the right-side term of (5.1) is the set

$$
\left\{\xi+\psi_{1} \cup \varphi+\psi_{m} \cup \varphi^{\prime} \mid \varphi, \varphi^{\prime} \in H^{1}\left(G, \mathbb{F}_{p}\right)\right\},
$$

and $\xi$ is any element of $\left\langle\psi_{1}, \ldots, \psi_{m}\right\rangle$; if $m=3$ then (5.1) is an equality. 


\subsection{Massey products and unipotent representations}

Massey products for a pro- $p$ group $G$ may be interpreted in terms of unipotent upper-triangular representations of $G$ as follows. For $m \geq 2$, let

$$
\mathbb{U}_{m+1}=\left\{\left(\begin{array}{ccccc}
1 & a_{1,2} & \cdots & & a_{1, m+1} \\
& 1 & a_{2,3} & \cdots & \\
& & \ddots & \ddots & \vdots \\
& & & 1 & a_{m, m+1} \\
& & & & 1
\end{array}\right) \mid a_{i, j} \in \mathbb{F}_{p}\right\} \subseteq \mathrm{GL}_{m+1}\left(\mathbb{F}_{p}\right)
$$

be the group of unipotent upper-triangular $(m+1) \times(m+1)$-matrices over $\mathbb{F}_{p}$. Then $\mathbb{U}_{m+1}$ is a $p$-group. Moreover, let $I_{m+1}, E_{i, j} \in \mathbb{U}_{m+1}$ denote respectively the identity $(m+1) \times(m+1)$-matrix and the $(m+1) \times(m+1)$-matrix with 1 at entry $(i, j)$ and 0 elsewhere, for $1 \leq i<j \leq m+1$.

Lemma 5.2. The group $\mathbb{U}_{m+1}$ has the following properties.

(i) The center $\mathrm{Z}\left(\mathbb{U}_{m+1}\right)$ is the subgroup

$$
I_{m+1}+E_{1, m+1} \mathbb{F}_{p}=\left\{I_{m+1}+a \cdot E_{1, m+1} \mid a \in \mathbb{F}_{p}\right\} .
$$

(ii) The exponent of $\mathbb{U}_{m+1}$ is $p^{\left\lfloor\log _{p}(m+1)\right\rfloor+1}$.

(iii) The $(m+1)$-th element $\gamma_{m+1}\left(\mathbb{U}_{m+1}\right)$ of the descending central series of $\mathbb{U}_{m+1}$ is trivial.

Proof. For (i), see, e.g., [24, § 3] or [12, p. 308]. For (ii), see [10, Proposition 2.3]. Item (iii) may be deduced from [24, Lemma 3.8].

One has the following group-theoretic result to check whether a given $m$-fold Massey product of a pro- $p$ group $G$ is defined or vanishes (e.g., [12, Lemma 9.3]).

Proposition 5.3. Let $G$ be a pro- $p$ group and $\psi_{1}, \ldots, \psi_{m} \in H^{1}\left(G, \mathbb{F}_{p}\right), m \geq 2$. Set $\overline{\mathbb{U}}_{m+1}=\mathbb{U}_{m+1} / Z\left(\mathbb{U}_{m+1}\right)$.

(i) The $m$-fold Massey product $\left\langle\psi_{1}, \ldots, \psi_{m}\right\rangle$ is defined if and only if there exists a continuous homomorphism $\bar{\rho}: G \rightarrow \overline{\mathbb{U}}_{m+1}$ such that $\bar{\rho}_{i, i+1}=\psi_{i}$ for every $i=1, \ldots, m$.

(ii) The $m$-fold Massey product $\left\langle\psi_{1}, \ldots, \psi_{m}\right\rangle$ vanishes if and only if there exists a continuous homomorphism $\rho: G \rightarrow \mathbb{U}_{m+1}$ such that $\rho_{i, i+1}=\psi_{i}$ for every $i=1, \ldots, m$.

(Here $\bar{\rho}_{i, i+1}$ and $\rho_{i, i+1}$ denote the projection of $\bar{\rho}$, resp. $\rho$, on the $(i, i+1)$-entry.) 
Let $\mathbb{K}$ be a field containing a primitive $p$-th root of 1 . In [23, Conjecture 1.1], Minac and Tân conjectured that every defined $m$-fold Massey product for every $m \geq 3$ on $H^{1}\left(G_{\mathbb{K}}(p), \mathbb{F}_{p}\right)$ vanishes. Roughly speaking, this conjecture says that if $\mathbb{K}$ is a field containing a primitive $p$-th root of 1 , the $\mathbb{F}_{p}$-cohomology algebra $H^{\bullet}\left(G_{\mathbb{K}}(p), \mathbb{F}_{p}\right)$ should allow no operations which are external with respect to its quadratic algebra structure given by the cup-product. This conjecture has been proved in the following cases:

(a) if $m=3$ by Efrat-Matzri, and independently by Minac-Tân (cf. [11,23]),

(b) if $m=4$ and $\mathbb{K}$ is a local field containing also a primitive $p^{2}$-th root of 1 by Guillot-Minac (cf. [16]),

(c) for every $m \geq 3$, if $\mathbb{K}$ is a number field by Harpaz-Wittenberg (cf. [18]).

In [24, §7], Minac and Tân produced some examples of pro- $p$ groups with defined and non-vanishing 3-fold Massey products, and hence which do not occur as maximal pro- $p$ Galois groups of fields containing a primitive $p$-th root of 1 .

Example 5.4. Let $G$ be the pro- $p$ group with minimal presentation

$$
G=\left\langle x_{1}, \ldots, x_{5} \mid\left[\left[x_{1}, x_{2}\right], x_{3}\right]\left[x_{4}, x_{5}\right]\right\rangle .
$$

Then there is a 3-fold Massey product on $H^{1}\left(G, \mathbb{F}_{p}\right)$ which is defined but does not vanish (cf. [24, Example 7.2]). Note that, by Proposition 2.1, the $\mathbb{F}_{p}$-cohomology algebra $H^{\bullet}\left(G, \mathbb{F}_{p}\right)$ is quadratic.

\subsection{The first type of $r_{0}$}

We split the proof of Theorem 1.2 (ii) (a) in the following two propositions.

Proposition 5.5. Let $G=\left\langle x_{1}, \ldots, x_{d} \mid r_{0}\right\rangle$ be a one-relator pro-p group with $r_{0}$ of the first type as defined in Theorem 1.1. Then every defined 3-fold Massey product in $H^{\bullet}\left(G, \mathbb{F}_{p}\right)$ vanishes if and only if $q \neq 3$.

Proof. Pick three elements $\psi_{1}, \psi_{2}, \psi_{3} \in H^{1}\left(G, \mathbb{F}_{p}\right)$ (by Proposition 5.1 (i), we may assume that they are all non-trivial), and write

$$
\psi_{1}=\sum_{i=1}^{d} a_{i} \chi_{i}, \quad \psi_{2}=\sum_{i=1}^{d} b_{i} \chi_{i}, \quad \psi_{3}=\sum_{i=1}^{d} c_{i} \chi_{i},
$$

with $a_{i}, b_{i}, c_{i} \in \mathbb{F}_{p}$. Then, by Proposition 2.1, one has

$$
\psi_{1} \cup H^{1}\left(G, \mathbb{F}_{p}\right)+\psi_{3} \cup H^{1}\left(G, \mathbb{F}_{p}\right)=H^{2}\left(G, \mathbb{F}_{p}\right) \ni 0,
$$

unless $a_{i}=c_{i}=0$ for every $i=2, \ldots, d$. 
Suppose that the Massey product $\left\langle\psi_{1}, \psi_{2}, \psi_{3}\right\rangle$ is defined. If some of the coefficients $a_{i}, c_{i}$ are not 0 for $i \in\{2, \ldots, d\}$, then by Proposition 5.1 (iii) and by (5.2), the Massey product $\left\langle\psi_{1}, \psi_{2}, \psi_{3}\right\rangle$ vanishes.

Assume that $q \neq 3$ and $a_{i}=c_{i}=0$ for every $i=2, \ldots, d$. Set

$$
A=I_{4}+a_{1} E_{1,2}+b_{1} E_{2,3}+c_{1} E_{3,4} \in \mathbb{U}_{4}, \quad B_{i}=I_{4}+b_{i} E_{2,3} \in \mathbb{U}_{4}
$$

for $i=2, \ldots, d$. By Lemma 5.2 (ii), the exponent of $\mathbb{U}_{4}$ is $p^{2}$ if $p=2,3$, and $p$ if $p \geq 5$. Therefore, the exponent of $\mathbb{U}_{4}$ divides $q$ (recall that 4 divides $q$ if $p=2$, and we are assuming now that $q \neq 3$ if $p=3$ ), and $A^{q}=I_{4}$. Also, by Lemma $5.2(\mathrm{c})$, one has $\gamma_{4}\left(\mathbb{U}_{4}\right)=\{1\}$. Finally, $\left[A,_{2} B_{2}\right]=\left[B_{i}, B_{i+1}\right]=I_{4}$ for every $2 \leq i \leq d-1, i$ even. Therefore,

$$
A^{q} \cdot\left[A,_{n} B_{2}\right] \cdot\left[B_{2}, B_{3}\right] \cdots\left[B_{d-1}, B_{d}\right]=I_{4}
$$

for any $n \geq 3$. Hence, the assignment $x_{1} \mapsto A, x_{i} \mapsto B_{i}$ for every $i=2, \ldots, d$ yields a homomorphism $\rho: G \rightarrow \mathbb{U}_{4}$, and thus $\left\langle\psi_{1}, \psi_{2}, \psi_{3}\right\rangle$ vanishes by Proposition 5.3 (ii).

Conversely, assume that $q=3$, and pick $\psi_{1}, \psi_{2}, \psi_{3} \in H^{1}\left(G, \mathbb{F}_{p}\right)$ such that

$$
a_{1}, b_{1}, c_{1} \neq 0 \quad \text { and } \quad a_{i}=c_{i}=0 \text { for } i=2, \ldots, d .
$$

For every matrix $A=\left(a_{h, k}\right) \in \mathbb{U}_{4}, B_{i}=\left(b(i)_{h, k}\right) \in \mathbb{U}_{4}$ such that

$$
\begin{aligned}
a_{1,2}=a_{1}, \quad a_{2,3} & =b_{1}, \quad a_{3,4}=c_{1}, \\
b(i)_{2,3} & =b_{i}, \quad b(i)_{1,2}=b(i)_{3,4}=0 \quad \text { for all } i=2, \ldots, d,
\end{aligned}
$$

one has

$$
\left[A,,_{2} B_{2}\right]=\left[B_{2}, B_{3}\right]=\cdots=\left[B_{d-1}, B_{d}\right]=I_{4} \quad \text { and } \quad A^{3}=I_{4}+a_{1} b_{1} c_{1} E_{1,4} .
$$

Therefore, equality (5.3) holds modulo $\mathrm{Z}\left(\mathbb{U}_{4}\right)$, and the assignment

$$
x_{1} \mapsto \bar{A}, \quad x_{i} \mapsto \bar{B}_{i} \quad \text { for every } i=2, \ldots, d
$$

(where $\bar{X}$ denotes the coset $X \cdot \mathrm{Z}\left(\mathbb{U}_{4}\right) \in \overline{\mathbb{U}}_{4}$ for $X \in \mathbb{U}_{4}$ ) yields a homomorphism $\bar{\rho}: G \rightarrow \overline{\mathbb{U}}_{4}$ which cannot lift to a homomorphism $\rho: G \rightarrow \mathbb{U}_{4}$ as $a_{1} b_{1} c_{1} \neq 0$. Thus, by Proposition 5.3, the Massey product $\left\langle\psi_{1}, \psi_{2}, \psi_{3}\right\rangle$ is defined but does not vanish.

Proposition 5.6. Let $G=\left\langle x_{1}, \ldots, x_{d} \mid r_{0}\right\rangle$ be a one-relator pro- $p$ group with $r_{0}$ of the first type as defined in Theorem 1.1. Then every defined 4-fold Massey product in $H^{\bullet}\left(G, \mathbb{F}_{p}\right)$ vanishes, unless $p=q=3$. 
Proof. Pick four elements $\psi_{1}, \psi_{2}, \psi_{3}, \psi_{4} \in H^{1}\left(G, \mathbb{F}_{p}\right)$ (by Proposition 5.1 (i), we may assume that they are all non-trivial), and write

$$
\psi_{1}=\sum_{i=1}^{d} a_{i} \chi_{i}, \quad \psi_{1}=\sum_{i=1}^{d} b_{i} \chi_{i}, \quad \psi_{3}=\sum_{i=1}^{d} c_{i} \chi_{i}, \quad \psi_{4}=\sum_{i=1}^{d} d_{i} \chi_{i},
$$

with $a_{i}, b_{i}, c_{i}, d_{i} \in \mathbb{F}_{p}$. Then

$$
\psi_{1} \cup H^{1}\left(G, \mathbb{F}_{p}\right)+\psi_{4} \cup H^{1}\left(G, \mathbb{F}_{p}\right)=H^{2}\left(G, \mathbb{F}_{p}\right) \ni 0,
$$

unless $a_{i}=d_{i}=0$ for every $i=2, \ldots, d$.

Suppose that the Massey product $\left\langle\psi_{1}, \psi_{2}, \psi_{3}, \psi_{4}\right\rangle$ is defined. If some of the coefficients $a_{i}, d_{i}$ are not 0 for $i \in\{2, \ldots, d\}$, then by Proposition 5.1 (iii) and by (5.4), the Massey product $\left\langle\psi_{1}, \ldots, \psi_{4}\right\rangle$ vanishes. Hence, we assume now that $\left\langle\psi_{1}, \psi_{2}, \psi_{3}, \psi_{4}\right\rangle$ is defined and that $a_{i}=d_{i}=0$ for every $i=2, \ldots, d$, while $a_{1}, d_{1} \neq 0$. By Proposition $5.3(\mathrm{i})$, one has

$$
\begin{aligned}
0=\psi_{2} \cup \psi_{3} & =\sum_{h=1}^{(d-1) / 2}\left(b_{2 h} c_{2 h+1}-b_{2 h+1} c_{2 h}\right) \chi_{2 h} \cup \chi_{2 h+1} \\
& =\left(\sum_{h=1}^{(d-1) / 2} b_{2 h} c_{2 h+1}-b_{2 h+1} c_{2 h}\right) \chi_{2} \cup \chi_{3},
\end{aligned}
$$

and therefore $\sum_{h} b_{2 h} c_{2 h+1}-b_{2 h+1} c_{2 h}=0$ - while both cup-products $\psi_{1} \cup \psi_{2}$ and $\psi_{3} \cup \psi_{4}$ are trivial because $\chi_{1} \cup H^{1}\left(G, \mathbb{F}_{p}\right)=0$. Henceforth, we assume $q \neq 3$, and we analyze the following two cases: (a) $n \geq 3$, and (b) $n=2$.

Case (a). Assume that $n \geq 3$, and set

$$
\begin{aligned}
A & =I_{5}+a_{1} E_{1,2}+b_{1} E_{2,3}+c_{1} E_{3,4}+d_{1} E_{4,5} \in \mathbb{U}_{5}, \\
B_{i} & =I_{5}+b_{i} E_{2,3}+c_{i} E_{3,4} \in \mathbb{U}_{5}
\end{aligned}
$$

for $i=2, \ldots, d$. By Lemma 5.2 (ii), the exponent of $\mathbb{U}_{5}$ is $p^{2}$, if $p=2,3$, and $p$ if $p \geq 5$. Therefore, the exponent of $\mathbb{U}_{5}$ divides $q$, and $A^{q}=I_{5}$. Moreover, one has $\left[A,_{3} B_{2}\right]=I_{5}$, while $\left[B_{i}, B_{i+1}\right]=I_{5}+\left(b_{i} c_{i+1}-b_{i+1} c_{i}\right) E_{2,4}$, and thus

$$
\prod_{h=1}^{(d-1) / 2}\left[B_{2 h}, B_{2 h+1}\right]=I_{5}+E_{2,4} \cdot \sum_{h=1}^{(d-1) / 2} b_{2 h} c_{2 h+1}-b_{2 h+1} c_{2 h} .
$$

Therefore, by (5.5), one has

$$
\begin{aligned}
A^{q} \cdot\left[A,{ }_{n} B_{2}\right] \cdot\left[B_{2}, B_{3}\right] \cdots\left[B_{d-1}, B_{d}\right] & =I_{5}, \\
\text { and also } \quad\left[\left[B_{i}, B_{j}\right],\left[B_{i^{\prime}}, B_{j^{\prime}}\right]\right] & =I_{5}
\end{aligned}
$$


for all $i, j, i^{\prime}, j^{\prime} \in\{2, \ldots, d\}$ so that the assignment

$$
x_{1} \mapsto A, \quad x_{i} \mapsto B_{i} \quad \text { for } i=2, \ldots, d
$$

yields a homomorphism $\rho: G \rightarrow \mathbb{U}_{5}$. Hence, the Massey product $\left\langle\psi_{1}, \psi_{2}, \psi_{3}\right\rangle$ vanishes by Proposition 5.3 (ii).

Case (b). Assume that $n=2$, and let $A, B_{i} \in \mathbb{U}_{5}$ be as above. If $b_{2}=0$ or $c_{2}=0$, then one has $\left[A, 2 B_{2}\right]=I_{5}$, and equality (5.6) holds also with $n=2$. Thus, the assignment $x_{1} \mapsto A, x_{i} \mapsto B_{i}$ for $i=2, \ldots, d$ yields a homomorphism $\rho: G \rightarrow \mathbb{U}_{5}$, and the Massey product $\left\langle\psi_{1}, \psi_{2}, \psi_{3}\right\rangle$ vanishes by Proposition 5.3 (ii). If $b_{2} \cdot c_{2} \neq 0$, then

$$
\left[A,{ }_{2} B_{2}\right]=I_{5}+a_{1} b_{2} c_{2} E_{1,4}+b_{2} c_{2} d_{1} E_{2,5} \neq I_{5} .
$$

Set $\tilde{B}_{3}=B_{3}-a_{1} b_{2} E_{1,3}+c_{2} d_{1} E_{3,4}$. Then

$$
\begin{aligned}
{\left[B_{2}, \tilde{B}_{3}\right] } & =I_{5}-a_{1} b_{2} c_{2} E_{1,4}+\left(b_{2} c_{3}-b_{3} c_{2}\right) E_{2,4}-b_{2} c_{2} d_{1} E_{2,5} \\
& =\left[A, 2 B_{2}\right]^{-1}+\left(b_{2} c_{3}-b_{3} c_{2}\right) E_{2,4},
\end{aligned}
$$

and equality (5.6) holds with $n=2$ and $\tilde{B}_{3}$ instead of $B_{3}$. Also, equality (5.7) holds after the replacement of $B_{3}$. Thus, the assignment $x_{1} \mapsto A, x_{3} \mapsto \tilde{B}_{3}, x_{i} \mapsto B_{i}$ for $i=2,4, \ldots, d$ yields a homomorphism $\rho: G \rightarrow \mathbb{U}_{5}$, and the Massey product $\left\langle\psi_{1}, \psi_{2}, \psi_{3}, \psi_{4}\right\rangle$ vanishes by Proposition 5.3 (ii).

\subsection{The second type of $\boldsymbol{r}_{0}$}

As for the first family of pro- $p$ groups, we split the proof of Theorem 1.2 (ii) (b) in the following two propositions.

Proposition 5.7. Let $G=\left\langle x_{1}, \ldots, x_{d} \mid r_{0}\right\rangle$ be a one-relator pro- $p$ group with $r_{0}$ of the second type as defined in Theorem 1.1. Then every defined 3-fold Massey product in $H^{\bullet}\left(G, \mathbb{F}_{p}\right)$ vanishes if and only if $q \neq 3$ and $n \geq 3$.

Proof. Pick three elements $\psi_{1}, \psi_{2}, \psi_{3} \in H^{1}\left(G, \mathbb{F}_{p}\right)$ (by Proposition 5.1 (i), we may assume that they are all non-trivial), and write

$$
\psi_{1}=\sum_{i=1}^{d} a_{i} \chi_{i}, \quad \psi_{1}=\sum_{i=1}^{d} b_{i} \chi_{i}, \quad \psi_{3}=\sum_{i=1}^{d} c_{i} \chi_{i},
$$

with $a_{i}, b_{i}, c_{i} \in \mathbb{F}_{p}$. Then, by Proposition 2.1, one has

$$
\psi_{1} \cup H^{1}\left(G, \mathbb{F}_{p}\right)+\psi_{3} \cup H^{1}\left(G, \mathbb{F}_{p}\right)=H^{2}\left(G, \mathbb{F}_{p}\right) \ni 0,
$$

unless $a_{i}=c_{i}=0$ for every $i=3, \ldots, d$. 
Suppose that the 3-fold Massey product $\left\langle\psi_{1}, \psi_{2}, \psi_{3}\right\rangle$ is defined. If some of the coefficients $a_{i}, c_{i}$ are not 0 for $i \in\{3, \ldots, d\}$, then by Proposition 5.1 (iii) and by (5.8), the Massey product $\left\langle\psi_{1}, \psi_{2}, \psi_{3}\right\rangle$ vanishes.

Assume that $q \neq 3, n \geq 3$, and let $\psi_{1}, \psi_{3}$ be such that $a_{i}=c_{i}=0$ for every $i=3, \ldots, d$. Set

$$
\begin{array}{ll}
A_{i}=I_{4}+a_{i} E_{1,2}+b_{i} E_{2,3}+c_{i} E_{3,4} \in \mathbb{U}_{4} & \text { for } i=1,2, \\
B_{i}=I_{4}+b_{i} E_{2,3} \in \mathbb{U}_{4} & \text { for } i=3, \ldots, d .
\end{array}
$$

By Lemma 5.2 (ii), the exponent of $\mathbb{U}_{4}$ is $p^{2}$ if $p=2,3$, and $p$ if $p \geq 5$. Therefore, the exponent of $\mathbb{U}_{4}$ divides $q$, and $A_{1}^{q}=I_{4}$. Also, by Lemma 5.2 (c), one has $\gamma_{4}\left(\mathbb{U}_{4}\right)=\{1\}$. Finally, one has $\left[B_{i}, B_{i+1}\right]=I_{4}$ for every $3 \leq i \leq d-1, i$ odd, and $\left[A_{1}, 3 A_{2}\right]=I_{3}$. Therefore, if $n \geq 3$, then

$$
A^{q} \cdot\left[A_{1, n} A_{2}\right] \cdot\left[B_{3}, B_{4}\right] \cdots\left[B_{d-1}, B_{d}\right]=I_{4},
$$

and the assignment $x_{i} \mapsto A_{i}$ and $x_{j} \mapsto B_{j}$ for $i=1,2$ and $j=3, \ldots, d$ yields a homomorphism $\rho: G \rightarrow \mathbb{U}_{4}$. Thus, $\left\langle\psi_{1}, \psi_{2}, \psi_{3}\right\rangle$ vanishes by Proposition 5.3 (ii).

Conversely, assume that $q=3$ or $n=2$, and let $\psi_{1}, \psi_{3}$ be such that $a_{i}=c_{i}=0$ for $i=3, \ldots, d$. For every matrix

$$
\begin{aligned}
& A_{i}=\left(a(i)_{h, k}\right) \in \mathbb{U}_{4}, \quad \text { with } i=1,2, \\
& B_{j}=\left(b(j)_{h, k}\right) \in \mathbb{U}_{4}, \quad \text { with } j=3, \ldots, d,
\end{aligned}
$$

such that

$$
\begin{aligned}
a(i)_{1,2}=a_{i}, \quad a_{2,3}(i)=b_{i}, & a(i)_{3,4}=c_{i}, \\
b(j)_{2,3}=b_{j}, & b(j)_{1,2}=b(i)_{3,4}=0 \quad \text { for all } i, j,
\end{aligned}
$$

one has

$$
\begin{aligned}
{\left[B_{j}, B_{j^{\prime}}\right] } & =I_{4} \quad \text { for every } j, j^{\prime} \in\{3, \ldots, d\}, \\
{\left[A_{1,2} A_{2}\right] } & =I_{4}+\left(a_{1} b_{2} c_{2}-2 a_{2} b_{1} c_{2}+a_{2} b_{2} c_{1}\right) E_{1,4}, \\
A_{1}^{3} & =I_{4}+a_{1} b_{1} c_{1} E_{1,4} .
\end{aligned}
$$

In this case, equality (5.3) holds modulo $\mathrm{Z}\left(\mathbb{U}_{4}\right)$, and the assignment

$$
x_{i} \mapsto \bar{A}_{i} \quad \text { and } \quad x_{j} \mapsto \bar{B}_{j} \quad \text { for every } i=1,2, j=3, \ldots, d
$$

(where $\bar{X}$ denotes the coset $X \cdot \mathrm{Z}\left(\mathbb{U}_{4}\right) \in \overline{\mathbb{U}}_{4}$, for $X \in \mathbb{U}_{4}$ ) yields a homomorphism $\bar{\rho}: G \rightarrow \overline{\mathbb{U}}_{4}$, and therefore, by Proposition 5.3 (i), the Massey product $\left\langle\psi_{1}, \psi_{2}, \psi_{3}\right\rangle$ is defined. One has two cases. 
(a) If $q=3$ and $a_{1} b_{1} c_{1} \neq 0$, and moreover

$$
a_{1} b_{2} c_{2}-2 a_{2} b_{1} c_{2}+a_{2} b_{2} c_{1} \neq-a_{1} b_{1} c_{1} \quad \text { if } n=2,
$$

then $\bar{\rho}$ cannot lift to a homomorphism $\rho: G \rightarrow \mathbb{U}_{4}$.

(b) Similarly, if $n=2$ and $a_{1} b_{2} c_{2}-2 a_{2} b_{1} c_{2}+a_{2} b_{2} c_{1} \neq 0$, and moreover

$$
a_{1} b_{1} c_{1} \neq-a_{1} b_{2} c_{2}+2 a_{2} b_{1} c_{2}-a_{2} b_{2} c_{1} \quad \text { if } q=3,
$$

then $\bar{\rho}$ cannot lift to a homomorphism $\rho: G \rightarrow \mathbb{U}_{4}$.

In both cases, Proposition 5.3 (ii) implies that $\left\langle\psi_{1}, \psi_{2}, \psi_{3}\right\rangle$ does not vanish.

Remark 5.8. If $n=2$, then one may deduce that $H^{\bullet}\left(G, \mathbb{F}_{p}\right)$ has defined Massey products which do not vanish also from [24, Theorem 7.12].

Proposition 5.9. Let $G=\left\langle x_{1}, \ldots, x_{d} \mid r_{0}\right\rangle$ be a one-relator pro- $p$ group with $r_{0}$ of the second type as defined in Theorem 1.1. If $q \neq 3$ and $n \geq 4$, then every defined 4-fold Massey product in $H^{\bullet}\left(G, \mathbb{F}_{p}\right)$ vanishes.

Proof. Pick four non-trivial elements $\psi_{1}, \psi_{2}, \psi_{3}, \psi_{4} \in H^{1}\left(G, \mathbb{F}_{p}\right)$, and write

$$
\psi_{1}=\sum_{i=1}^{d} a_{i} \chi_{i}, \quad \psi_{1}=\sum_{i=1}^{d} b_{i} \chi_{i}, \quad \psi_{3}=\sum_{i=1}^{d} c_{i} \chi_{i}, \quad \psi_{4}=\sum_{i=1}^{d} d_{i} \chi_{i},
$$

with $a_{i}, b_{i}, c_{i}, d_{i} \in \mathbb{F}_{p}$. Then

$$
\psi_{1} \cup H^{1}\left(G, \mathbb{F}_{p}\right)+\psi_{4} \cup H^{1}\left(G, \mathbb{F}_{p}\right)=H^{2}\left(G, \mathbb{F}_{p}\right) \ni 0,
$$

unless $a_{i}=d_{i}=0$ for every $i=3, \ldots, d$.

Suppose that the Massey product $\left\langle\psi_{1}, \psi_{2}, \psi_{3}, \psi_{4}\right\rangle$ is defined. If some of the coefficients $a_{i}, d_{i}$ are not 0 for $i \in\{3, \ldots, d\}$, then by Proposition 5.1 (iii) and by (5.9), the Massey product $\left\langle\psi_{1}, \ldots, \psi_{4}\right\rangle$ vanishes. Hence, we assume now that $\left\langle\psi_{1}, \psi_{2}, \psi_{3}, \psi_{4}\right\rangle$ is defined and that $a_{i}=d_{i}=0$ for every $i=3, \ldots, d$.

By Proposition 5.3 (i), one has

$$
\begin{aligned}
0=\psi_{2} \cup \psi_{3} & =\sum_{h=2}^{d / 2}\left(b_{2 h-1} c_{2 h}-b_{2 h} c_{2 h-1}\right) \chi_{2 h-1} \cup \chi_{2 h} \\
& =\left(\sum_{h=1}^{(d-1) / 2} b_{2 h} c_{2 h+1}-b_{2 h} c_{2 h-1}\right) \chi_{3} \cup \chi_{4},
\end{aligned}
$$


and therefore $\sum_{h} b_{2 h-1} c_{2 h}-b_{2 h} c_{2 h-1}=0$ - while both cup-products $\psi_{1} \cup \psi_{2}$ and $\psi_{3} \cup \psi_{4}$ are trivial because $\chi_{1} \cup H^{1}\left(G, \mathbb{F}_{p}\right)=\chi_{2} \cup H^{1}\left(G, \mathbb{F}_{p}\right)=0$.

Assume that $n \geq 4$, and set

$$
\begin{aligned}
& A_{i}=I_{4}+a_{i} E_{1,2}+b_{i} E_{2,3}+c_{i} E_{3,4}+d_{i} E_{4,5} \in \mathbb{U}_{5}, \\
& B_{j}=I_{4}+b_{j} E_{2,3}+c_{j} E_{3,4} \in \mathbb{U}_{5}
\end{aligned}
$$

for $i=1,2$ and $j=3, \ldots, d$. By Lemma 5.2 (ii), the exponent of $\mathbb{U}_{5}$ is $p^{2}$ if $p=2,3$, and $p$ if $p \geq 5$. Therefore, the exponent of $\mathbb{U}_{5}$ divides $q$, and $A_{1}^{q}=I_{5}$. Moreover, one has $\left[A_{1,4} A_{2}\right]=I_{5}$ and $\left[B_{j}, B_{j+1}\right]=I_{5}+\left(b_{j} c_{j+1}-b_{j+1} c_{j}\right) E_{2,4}$ so that

$$
\prod_{h=2}^{d / 2}\left[B_{2 h-1}, B_{2 h}\right]=I_{5}+E_{2,4} \cdot \sum_{h=2}^{d / 2} b_{2 h-1} c_{2 h}-b_{2 h} c_{2 h-1} .
$$

Therefore, by (5.10), one has

$$
\begin{aligned}
A^{q} \cdot\left[A_{1, n} A_{2}\right] \cdot\left[B_{3}, B_{4}\right] \cdots\left[B_{d-1}, B_{d}\right] & =I_{5}, \\
\text { and also } \quad\left[\left[B_{i}, B_{j}\right],\left[B_{i^{\prime}}, B_{j^{\prime}}\right]\right] & =I_{5}
\end{aligned}
$$

for every $i, j, i^{\prime}, j^{\prime} \in\{3, \ldots, d\}$ so that the assignment $x_{i} \mapsto A_{i}, x_{j} \mapsto B_{j}$ for $i=1,2, j=3, \ldots, d$ yields a homomorphism $\rho: G \rightarrow \mathbb{U}_{5}$. Hence, the Massey product $\left\langle\psi_{1}, \psi_{2}, \psi_{3}, \psi_{4}\right\rangle$ vanishes by Proposition 5.3 (ii).

Remark 5.10. If $n=3$, then the vanishing of 4-fold Massey products depends also on $d$. For example, the Massey product $\left\langle\chi_{1}, \chi_{2}+\chi_{3}, \chi_{2}+\chi_{3}, \chi_{2}\right\rangle$ is always defined and does not vanish if $d=4$, while it vanishes if $d \geq 6$.

We conclude with the following observation.

Remark 5.11. From the two families of pro- $p$ groups defined in Theorem 1.1, one may produce several further examples of pro- $p$ groups with more than one relation, whose $\mathbb{F}_{p}$-cohomology algebra is quadratic, for example among mild pro- $p$ groups - e.g., if

$$
G=\left\langle x_{1}, \ldots, x_{d} \mid r_{0}, r_{1}\right\rangle, \quad \text { with } r_{1}=\left[x_{d-2}, x_{d-1}\right] \cdot \prod_{i, j}\left[x_{i}, x_{j}\right]
$$

for some $3 \leq i<j \leq d$, then $G$ is mild and $H^{\bullet}\left(G, \mathbb{F}_{p}\right)$ is quadratic, cf. [31, Proposition 3.6]. It may be interesting to investigate the behavior of these pro- $p$ groups with respect to 3- and 4-fold Massey products and free and abelian subgroups in order to obtain results similar to Theorem 1.2 (ii) (a)-(b) and Proposition 2.6. 
Acknowledgments. The author is grateful to N. D. Tân and J. Minac for the inspiring discussions on J. P. Labute's article [20], I. Efrat for the investigation on the Kummerian property of pro- $p$ groups (a fundamental ingredient for this paper) pursued in [12], I. Snopce for the stimulating discussions on pro- $p$ groups and absolute Galois groups of fields, F. Matucci and T. S. Weigel for their useful comments, and the anonymous referee for her/his careful review and suggestions. Moreover, the author wishes to remark that the research carried out in the current work (and also in past papers) has been deeply influenced by J. P. Labute's life-time work on pro- $p$ groups and their cohomology.

\section{Bibliography}

[1] E. Becker, Euklidische Körper und euklidische Hüllen von Körpern, J. Reine Angew. Math. 268(269) (1974), 41-52.

[2] D. Benson, N. Lemire, J. Mináč and J. Swallow, Detecting pro- $p$-groups that are not absolute Galois groups, J. Reine Angew. Math. 613 (2007), 175-191.

[3] S. K. Chebolu, I. Efrat and J. Mináč, Quotients of absolute Galois groups which determine the entire Galois cohomology, Math. Ann. 352 (2012), no. 1, 205-221.

[4] C. De Clercq and M. Florence, Lifting theorems and smooth profinite groups, preprint (2017), https://arxiv.org/abs/1710.10631.

[5] J.D. Dixon, M. P.F. du Sautoy, A. Mann and D. Segal, Analytic pro-p groups, 2nd ed., Cambridge Stud. Adv. Math. 61, Cambridge University, Cambridge, 1999.

[6] W. G. Dwyer, Homology, Massey products and maps between groups, J. Pure Appl. Algebra 6 (1975), no. 2, 177-190.

[7] I. Efrat, Small maximal pro- $p$ Galois groups, Manuscripta Math. 95 (1998), no. 2, 237-249.

[8] I. Efrat, Valuations, Orderings, and Milnor K-Theory, Math. Surveys Monogr. 124, American Mathematical Society, Providence, 2006.

[9] I. Efrat, The Zassenhaus filtration, Massey products, and representations of profinite groups, Adv. Math. 263 (2014), 389-411.

[10] I. Efrat, The lower $p$-central series of a free profinite group and the shuffle algebra, J. Pure Appl. Algebra 224 (2020), no. 6, Article ID 106260.

[11] I. Efrat and E. Matzri, Triple Massey products and absolute Galois groups, J. Eur. Math. Soc. (JEMS) 19 (2017), no. 12, 3629-3640.

[12] I. Efrat and C. Quadrelli, The Kummerian property and maximal pro- $p$ Galois groups, J. Algebra 525 (2019), 284-310.

[13] A. J. Engler and J. Koenigsmann, Abelian subgroups of pro- $p$ Galois groups, Trans. Amer. Math. Soc. 350 (1998), no. 6, 2473-2485. 
[14] A. J. Engler and J. A. B. Nogueira, Maximal abelian normal subgroups of Galois pro-2-groups, J. Algebra 166 (1994), no. 3, 481-505.

[15] W.-D. Geyer, Field theory, in: Proceedings of the Winter School on Galois Theory, Trav. Math. 22, University of Luxembourg, Luxembourg (2013), 5-177.

[16] P. Guillot and J. Mináč, Extensions of unipotent groups, Massey products and Galois theory, Adv. Math. 354 (2019), Article ID 106748.

[17] P. Guillot, J. Mináč and A. Topaz, Four-fold Massey products in Galois cohomology, Compos. Math. 154 (2018), no. 9, 1921-1959.

[18] Y. Harpaz and O. Wittenberg, The Massey vanishing conjecture for number fields, preprint (2019), https://arxiv.org/abs/1904.06512.

[19] D. Kraines, Massey higher products, Trans. Amer. Math. Soc. 124 (1966), 431-449.

[20] J. P. Labute, Classification of Demushkin groups, Canadian J. Math. 19 (1967), 106132.

[21] J. Mináč, F. Pop, A. Topaz and K. Wickelgren, Nilpotent fundamental groups, Report of the workshop "Nilpotent Fundamental Groups", Banff AB, Banff, 2017.

[22] J. Mináč, M. Rogelstad and N. D. Tân, Relations in the maximal pro- $p$ quotients of absolute Galois groups, Trans. Amer. Math. Soc. 373 (2020), no. 4, 2499-2524.

[23] J. Mináč and N. D. Tân, Triple Massey products vanish over all fields, J. Lond. Math. Soc. (2) 94 (2016), no. 3, 909-932.

[24] J. Mináč and N. D. Tân, Triple Massey products and Galois theory, J. Eur. Math. Soc. (JEMS) 19 (2017), no. 1, 255-284.

[25] J. Neukirch, A. Schmidt and K. Wingberg, Cohomology of Number Fields, Grundlehren Math. Wiss. 323, Springer, Berlin, 2000.

[26] A. Polishchuk and L. Positselski, Quadratic Algebras, Univ. Lecture Ser. 37, American Mathematical Society, Providence, 2005.

[27] C. Quadrelli, Bloch-Kato pro- $p$ groups and locally powerful groups, Forum Math. 26 (2014), no. 3, 793-814.

[28] C. Quadrelli, 1-smooth pro- $p$ groups and Bloch-Kato pro- $p$ groups, preprint (2019), https://arxiv.org/abs/1904.00667.

[29] C. Quadrelli, Chasing maximal pro- $p$ Galois groups with 1-cyclotomicity, preprint (2021), https://arxiv.org/abs/2106.00335.

[30] C. Quadrelli, One-relator maximal pro- $p$ Galois groups and the Koszulity conjectures, Q. J. Math. (2020), DOI 10.1093/qmath/haaa049.

[31] C. Quadrelli, Pro- $p$ groups with few relations and universal Koszulity, Math. Scand. 127 (2021), no. 1, 28-42.

[32] C. Quadrelli, I. Snopce and M. Vannacci, On pro- $p$ groups with quadratic cohomology, preprint (2019), https://arxiv.org/abs/1906.04789. 
[33] C. Quadrelli and T. S. Weigel, Profinite groups with a cyclotomic $p$-orientation, Doc. Math. 25 (2020), 1881-1916.

[34] J.-P. Serre, Galois Cohomology, Springer Monogr. Math., Springer, Berlin, 2002.

[35] J.-P. Serre, Topics in Galois Theory, 2nd ed., Res. Notes Math. 1, A K Peters, Wellesley, 2008.

[36] I. Snopce and P. A. Zalesskii, Right-angled Artin pro- $p$-groups, preprint (2020), https://arxiv.org/abs/2005.01685; to appear in Selecta. Math. (N. S.).

[37] V. Voevodsky, On motivic cohomology with Z/l-coefficients, Ann. of Math. (2) 174 (2011), no. 1, 401-438.

[38] D. Vogel, Massey products in the galois cohomology of number fields, $\mathrm{PhD}$ thesis, University of Heidelberg, 2004.

[39] R. Ware, Galois groups of maximal p-extensions, Trans. Amer. Math. Soc. 333 (1992), no. 2, 721-728.

[40] C. Weibel, 2007 Trieste lectures on the proof of the Bloch-Kato conjecture, in: Some Recent Developments in Algebraic K-Theory, ICTP Lect. Notes 23, Abdus Salam International Centre for Theoretical Physics, Trieste (2008), 277-305.

[41] C. Weibel, The norm residue isomorphism theorem, J. Topol. 2 (2009), no. 2, 346372 .

[42] T. Würfel, On a class of pro- $p$ groups occurring in Galois theory, J. Pure Appl. Algebra 36 (1985), no. 1, 95-103.

Received November 20, 2020; revised April 3, 2021.

\section{Author information}

Corresponding author:

Claudio Quadrelli, Department of Mathematics and Applications,

University of Milano Bicocca, 20125 Milan, Italy.

E-mail: claudio.quadrelli@unimib.it 